\title{
System of fractional boundary value problem with $p$-Laplacian and advanced arguments
}

\author{
Amina Mahdjouba', Juan J. Nieto ${ }^{2 *}$ (1) and Abdelghani Ouahab'
}

\section{"Correspondence:}

juanjose.nieto.roig@usc.es

${ }^{2}$ Departamento de Estatística,

Análise Matemática, e Optimización,

Instituto de Matemáticas,

Universidade de Santiago de

Compostela, Santiago de

Compostela, 15782, Spain

Full list of author information is

available at the end of the article

\begin{abstract}
In this paper, we discuss the existence and multiplicity of positive solutions for a system of fractional differential equations with boundary condition and advanced arguments. The existence result is proved via Leray-Schauder's fixed point theorem type in a vector Banach space. Further, by using a new fixed point theorem in order Banach space, we study the multiplicity of positive solutions. Finally, some examples are given to illustrate our results.
\end{abstract}

MSC: 34A08; 34B15; 34B18

Keywords: Fractional differential equations; $p$-Laplacian operator; Cone; Fixed point theorem; Positive solutions; Multiplicity of solutions

\section{Introduction}

Fractional calculus and differential equations have now proved to be important tools modeling many real world phenomena like chemistry and physics [11, 22, 23, 25]). For the description of hereditary properties of fractional calculus, see $[20,24,32,37]$ and the references therein.

Recently, there have been some papers dealing with the existence and multiplicity of solution (or positive solution) of nonlinear initial fractional differential equation by the use of techniques of nonlinear analysis, see [2-7, 9, 33, 35, 38].

For example, Chai obtained in [10] the existence of at least one nonnegative solution and two positive solutions by using fixed point theorem on cone for the following problem:

$$
\left\{\begin{array}{l}
\left(\varphi_{p}\left(u^{\prime}(t)\right)^{\prime}+a(t) f(u(t))=0, \quad 0<t<1,\right. \\
u(0)=u^{\prime}(1)=0
\end{array}\right.
$$

Su et al. [31] studied the existence of one and two positive solutions by using the fixed point index theory of the following boundary values problems:

$$
\left\{\begin{array}{l}
\left(\varphi_{p}\left(u^{\prime}(t)\right)^{\prime}+a(t) f(u(t))=0, \quad 0<t<1,\right. \\
\alpha \varphi_{p}(u(0))-\beta \varphi_{p}\left(u^{\prime}(\xi)\right)=0, \quad \gamma \varphi_{p}(u(1))+\delta \varphi_{p}\left(u^{\prime}(\eta)\right)=0 .
\end{array}\right.
$$

(c) The Author(s) 2021. This article is licensed under a Creative Commons Attribution 4.0 International License, which permits use, sharing, adaptation, distribution and reproduction in any medium or format, as long as you give appropriate credit to the original author(s) and the source, provide a link to the Creative Commons licence, and indicate if changes were made. The images or other third party material in this article are included in the article's Creative Commons licence, unless indicated otherwise in a credit line to the material. If material is not included in the article's Creative Commons licence and your intended use is not permitted by statutory regulation or exceeds the permitted use, you will need to obtain permission directly from the copyright holder. To view a copy of this licence, visit http://creativecommons.org/licenses/by/4.0/. 
Tang et al. [34] studied the existence of positive solutions of fractional differential equation with $p$-Laplacian of the following type (1.3) by using the coincidence degree theory.

$$
\left\{\begin{array}{l}
D_{0^{+}}^{\alpha}\left(\phi\left(D_{0^{+}}^{\beta} u(t)\right)\right)(t)=f\left(t, u(t), D_{0^{+}}^{\beta} u(t)\right), \quad 0<t<1, \\
D_{0^{+}}^{\alpha} u(0)=0, \quad D_{0^{+}}^{\beta} u(1)=\gamma D_{0^{+}}^{\beta} u(1) .
\end{array}\right.
$$

In this work, we study the existence and multiplicity of positive solutions of the following problem:

$$
\left\{\begin{array}{l}
\left(\varphi_{p}\left(D_{0^{+}}^{\alpha} u(t)\right)\right)^{\prime}+a_{1}(t) f\left(u\left(\theta_{1}(t)\right), v\left(\theta_{2}(t)\right)\right)=0, \quad 0<t<1, \\
\left(\varphi_{\tilde{p}}\left(D_{0^{+}}^{\alpha} \nu(t)\right)\right)^{\prime}+a_{2}(t) g\left(u\left(\theta_{1}(t)\right), v\left(\theta_{2}(t)\right)\right)=0, \quad 0<t<1, \\
D_{0^{+}}^{\alpha} u(0)=u(0)=u^{\prime}(0)=0, \quad D_{0^{+}}^{\beta} u(1)=\gamma D_{0^{+}}^{\beta} u(\eta), \\
D_{0^{+}}^{\alpha} \nu(0)=v(0)=v^{\prime}(0)=0, \quad D_{0^{+}}^{\beta} \nu(1)=\gamma D_{0^{+}}^{\beta} \nu(\eta),
\end{array}\right.
$$

where $\eta \in(0,1), \gamma \in\left(0, \frac{1}{\eta^{\alpha-\beta-1}}\right), D_{0^{+}}^{\alpha}, D_{0^{+}}^{\beta}$ are the standard Riemann-Liouville fractional derivatives with $\alpha \in(2,3), \beta \in(1,2)$ such that $\alpha \geq \beta+1, p$-Laplacian operator is defined as $\varphi_{p}(s)=|s|^{p-2} s, p>1$, and the functions $f, g \in C\left(\mathbb{R}^{2}, \mathbb{R}\right)$.

In recent years, many authors studied the existence of solutions for systems of difference and differential equations with and without fractional derivative by using the vector version of the fixed point theorem (see [1, 8, 13, 15-19, 21, 26-28], the monograph of Graef et al. [12], and the references therein).

For establishing the existence and multiplicity of positive solutions of problem (1.4), let us list the following assumptions:

$\left(H_{1}\right) a_{i} \in L^{1}[0,1]$ is nonnegative and $a_{i}(t) \not \equiv 0$ on any subinterval of $[0,1]$ for $i=1,2$.

$\left(H_{2}\right)$ The advanced argument $\theta \in C((0,1),(0,1])$ and $0 \leq \theta(t) \leq 1, \forall t \in(0,1)$.

This work is organized as follows: In Sect. 2, we introduce all the background material used in this paper such as fractional calculus analysis and some results from fixed point theory. In Sects. 3, 4, the existence and multiplicity results of solutions for a system of fractional $p$-Laplace differential equations (1.4) are discussed by using the fixed point theorems in the generalized Banach space. We end the paper with two examples to illustrate our main results.

\section{Preliminaries}

In this section, we introduce some preliminary facts which are used throughout this paper.

Definition 2.1 ([14]) Let $X$ be a real Banach space. A nonempty closed convex set $P \subset X$ is called cone if

(1) $x \in P, \lambda \geq 0$, then $\lambda x \in P$;

(2) $x \in P,-x \in P$, then $x=0$.

If $x, y \in \mathbb{R}^{n}$ with $x=\left(x_{1}, \ldots, x_{n}\right)$ and $y=\left(y_{1}, \ldots, y_{n}\right)$, then by $x \leq y$ we mean $x_{i} \leq y_{i}$ for all $i=1, \ldots, n$. Also we set $|x|=\left(\left|x_{1}\right|, \ldots,\left|x_{n}\right|\right), \max (x, y)=\left(\max \left(x_{1}, y_{1}\right), \ldots, \max \left(x_{n}, y_{n}\right)\right)$, and $\mathbb{R}_{+}^{n}=\left\{x \in \mathbb{R}^{n}: x_{i}>0\right\}$. If $c \in \mathbb{R}$, then $x \leq c$ means $x_{i} \leq c$ for each $i=1, \ldots, n$.

Definition 2.2 Let $X$ be a nonempty set, and consider the space $\mathbb{R}_{+}^{m}$ endowed with the usual component-wise partial order. The mapping $d: X \times X \rightarrow \mathbb{R}_{+}^{m}$, which satisfies all the 
usual axioms of the metric, is called a generalized metric in Perov's sense and $(X, d)$ is called a generalized metric space.

Let $(X, d)$ be a generalized metric space in Perov's sense. For $r:=\left(r_{1}, \ldots, r_{m}\right) \in \mathbb{R}_{+}^{m}$, we will denote by

$$
B\left(x_{0}, r\right)=\left\{x \in X: d\left(x_{0}, x\right)<r\right\}
$$

the open ball centered in $x_{0}$ with radius $r$, and by

$$
\overline{B\left(x_{0}, r\right)}=\left\{x \in X: d\left(x_{0}, x\right) \leq r\right\}
$$

the closed ball centered at $x_{0}$ with radius $r$.

Theorem 2.1 ([12,36]) Let $X$ be a generalized Banach space, and let $N: X \rightarrow X$ be a completely continuous operator. Then either

(i) the equation $N(x)=x$ has at least one solution, or

(ii) the set $\mathcal{M}=\{x \in X \mid \mu N(x)=x, \mu \in(0,1)\}$ is unbounded.

Theorem 2.2 ([30]) Let $(X,\|\cdot\|)$ be a normed space, $P_{1}, P_{2} \subset X$ be two cones; $P:=$ $P_{1} \times P_{2} ; r, R \in \mathbb{R}_{+}^{2}, P_{r, R}:=\left\{u \in P_{i}: r_{i} \leq\left\|u_{i}\right\| \leq R_{i}\right\}$ with $0<r<R$; and let $N: P_{r, R} \rightarrow P, N=$ $\left(N_{1}, N_{2}\right)$ be a compact map. Assume that, for each $i \in\{1,2\}$, one of the following conditions is satisfied in $P_{r, R}$ :

(1) $N_{i}\left(u_{i}\right) \nprec u_{i}$ if $\left\|u_{i}\right\|=r_{i}$, and $N_{i}\left(u_{i}\right) \nsucc u_{i}$ if $\left\|u_{i}\right\|=R_{i}$;

(2) $N_{i}\left(u_{i}\right) \nsucc u_{i}$ if $\left\|u_{i}\right\|=r_{i}$, and $N_{i}\left(u_{i}\right) \nprec u_{i}$ if $\left\|u_{i}\right\|=R_{i}$.

Then $N$ has a fixed point $u$ in $P$ with $r_{i} \leq\left\|u_{i}\right\| \leq R_{i}$ for $i \in\{1,2\}$, where $\preceq$, namely $u \preceq v$ if and only if $v-u \in P$. We shall say that $u \prec v$ if $v-u \in P \backslash\{0\}$.

Remark 2.1 ([30]) In Theorem (2.2) four cases are possible for $u \in p_{r, R}$ :

$\left(c_{1}\right) N_{1}(u) \nprec u_{1}$ if $\left\|u_{1}\right\|=r_{1}$, and $N_{1}(u) \nsucc u_{1}$ if $\left\|u_{1}\right\|=R_{1}, N_{2}(u) \nprec u_{2}$ if $\left\|u_{2}\right\|=r_{2}$, and $N_{2}(u) \nsucc u_{2}$ if $\left\|u_{2}\right\|=R_{2}$;

(c. $N_{2}(u) \nprec u_{1}$ if $\left\|u_{1}\right\|=r_{1}$, and $N_{1}(u) \nsucc u_{1}$ if $\left\|u_{1}\right\|=R_{1}, N_{2}(u) \nsucc u_{2}$ if $\left\|u_{2}\right\|=r_{2}$, and $N_{2}(u) \nprec u_{2}$ if $\left\|u_{2}\right\|=R_{2}$;

$\left(c_{3}\right) N_{1}(u) \nsucc u_{1}$ if $\left\|u_{1}\right\|=r_{1}$, and $N_{1}(u) \nprec u_{1}$ if $\left\|u_{1}\right\|=R_{1}, N_{2}(u) \nprec u_{2}$ if $\left\|u_{2}\right\|=r_{2}$, and $N_{2}(u) \nsucc u_{2}$ if $\left\|u_{2}\right\|=R_{2}$;

$\left(c_{4}\right) N_{1}(u) \nsucc u_{1}$ if $\left\|u_{1}\right\|=r_{1}$, and $N_{1}(u) \nprec u_{1}$ if $\left\|u_{1}\right\|=R_{1}, N_{2}(u) \nsucc u_{2}$ if $\left\|u_{2}\right\|=r_{2}$, and $N_{2}(u) \nprec u_{2}$ if $\left\|u_{2}\right\|=R_{2}$.

Theorem 2.3 ([29]) Let $(X,\|\cdot\|)$ be a Banach space, $P_{1}, P_{2} \subset X$ be two cones, and $P:=$ $P_{1} \times P_{2}$ be the corresponding cone of $X^{2}=X \times X$, and let $\alpha_{i}, \beta_{i}>0$. We denote

$$
U_{\alpha_{i}}=\left\{u \in P_{i}:\|u\|<\alpha_{i}\right\} \quad \text { and } \quad V_{\beta_{i}}=\left\{u \in P_{i}:\|u\|<\beta_{i}\right\} \text {, }
$$

with $\alpha_{i} \neq \beta_{i}, r_{i}=\min \left\{\alpha_{i}, \beta_{i}\right\}$ and $R_{i}=\max \left\{\alpha_{i}, \beta_{i}\right\}$ for $i=1,2$. Assume that $N: \overline{W_{1} \times W_{2}} \rightarrow$ $P, N=\left(N_{1}, N_{2}\right)$ is a compact map (where $W_{i}=U_{\alpha_{i}} \cup V_{\beta_{i}}$ for $\left.i=1,2\right)$ and there exist $h_{i} \in$ $P_{i} \backslash\{0\}, i=1,2$, such that for each $i \in\{1,2\}$ the following condition is satisfied in $\overline{W_{1} \times W_{2}}$ :

$$
\lambda u_{i} \neq N_{i} u \quad \text { for }\left\|u_{i}\right\|=\alpha_{i} \text { and } \lambda \geq 1 ;
$$




$$
u_{i} \neq N_{i} u+\mu h_{i} \quad \text { for }\left\|u_{i}\right\|=\beta_{i} \text { and } \mu \geq 0 \text {. }
$$

Then

(1) $N$ has at least one fixed point $u=\left(u_{1}, u_{2}\right)$ in $P$ such that $u_{i} \in U_{\alpha_{i}} \backslash \overline{V_{\beta_{i}}}$ for $i=1,2$ if $\alpha_{i}>\beta_{i}$ for $i=1,2$;

(2) $N$ has at least two fixed points located in $\left(U_{\alpha_{1}} \backslash \overline{V_{\beta_{1}}}\right) \times U_{\alpha_{2}}$ and $\left(U_{\alpha_{1}} \backslash \overline{V_{\beta_{1}}}\right) \times\left(V_{\beta_{2}} \backslash U_{\alpha_{2}}\right)$ if $\beta_{1}<\alpha_{1}$ and $\beta_{2}>\alpha_{2} ;$

(3) $N$ has at least two fixed points located in $U_{\alpha_{1}} \times\left(U_{\alpha_{2}} \backslash \overline{V_{\beta_{2}}}\right)$ and $\left(V_{\beta_{1}} \backslash \overline{U_{\alpha_{1}}}\right) \times\left(U_{\alpha_{2}} \backslash \overline{V_{\beta_{2}}}\right)$ if $\beta_{1}>\alpha_{1}$ and $\beta_{2}<\alpha_{2}$;

(4) $N$ has at least four (three nontrivial) fixed points in $U_{\alpha_{1}} \times U_{\alpha_{2}}, U_{\alpha_{1}} \times\left(V_{\beta_{2}} \backslash \overline{U_{\alpha_{2}}}\right),\left(V_{\beta_{1}} \backslash \overline{U_{\alpha_{1}}}\right) \times U_{\alpha_{2}}$, and $\left(V_{\beta_{1}} \backslash \overline{U_{\alpha_{1}}}\right) \times\left(V_{\beta_{2}} \backslash \overline{U_{\alpha_{2}}}\right)$ if $\alpha_{i}<\beta_{i}$ for $i=1,2$.

Remark 2.2 ([29]) Our previous results can be easily generalized to systems of $n$ operator equations.

Definition 2.3 ([7]) The fractional integral of Riemann-Liouville of the function $h \in$ $L^{1}((0, \infty), \mathbb{R})$ of order $\alpha>0$ is defined by

$$
I_{0^{+}}^{\alpha} h(t)=\frac{1}{\Gamma(\alpha)} \int_{0}^{t}(t-s)^{\alpha-1} h(s) d s,
$$

where $\Gamma(\alpha)$ is the Euler gamma function defined by

$$
\Gamma(\alpha)=\int_{0}^{\infty} t^{\alpha-1} e^{-t} d t
$$

Definition 2.4 For a function $h \in A C^{n}(J)$, the Riemann-Liouville fractional order derivative of order $\alpha>0$ of $h$ is defined by

$$
D_{0^{+}}^{\alpha} h(t)=\frac{1}{\Gamma(n-\alpha)} \frac{d^{n}}{d t^{n}} \int_{0}^{t} \frac{h(s)}{(t-s)^{\alpha-n+1}} d s,
$$

where $n=[\alpha]+1$ and $[\alpha]$ denotes the integer part of the real number $\alpha$.

\section{Remark 2.3 ([7])}

(1) If $\lambda>-1$

$$
D_{0^{+}}^{\alpha} t^{\lambda}=\frac{\Gamma(\lambda+1)}{\Gamma(\lambda-\alpha+1} t^{\lambda-\alpha},
$$

and $D_{0^{+}}^{\alpha} t^{\alpha-m}=0, m=1,2, \ldots, n$, where $n=[\alpha]+1$.

(2) $D_{0^{+}}^{\alpha} I_{0^{+}}^{\alpha} u(t)=u(t)$ for all $u \in C(0,1) \cap L^{1}(0,1)$.

(3) If $u \in L^{1}(0,1), \alpha>\beta>0$, then

$$
D_{0^{+}}^{\beta} I_{0^{+}}^{\alpha} u(t)=I_{0^{+}}^{\alpha-\beta} u(t)
$$

Lemma 2.1 ([7]) If we assume that $u \in C(0,1) \cap L^{1}(0,1)$, then the fractional differential equation

$$
D_{0^{+}}^{\alpha} u(t)=0, \quad \alpha>0
$$


has $u(t)=C_{1} t^{\alpha-1}+C_{2} t^{\alpha-2}+\cdots+C_{n} t^{\alpha-n}, C_{i} \in \mathbb{R}, i=1,2, \ldots, n$, as a unique solution, where $n=[\alpha]+1$.

Lemma 2.2 ([7]) Suppose that $u \in C(0,1) \cap L^{1}(0,1)$ is such that $D_{0^{+}}^{\alpha} u \in C(0,1) \cap L^{1}(0,1)$.

Then

$$
I_{0^{+}}^{\alpha} D_{0^{+}}^{\alpha} u(t)=u(t)+C_{1} t^{\alpha-1}+C_{2} t^{\alpha-2}+\cdots+C_{n} t^{\alpha-n}
$$

for some $C_{i} \in \mathbb{R}, i=1,2, \ldots, n$, where $n=[\alpha]+1$.

Lemma 2.3 ([10]) If $x, y \geq 0, \gamma>0$, then

$$
(x+y)^{\gamma} \leq \max \left\{2^{\gamma-1}, 1\right\}\left(x^{\gamma}+y^{\gamma}\right) \text {. }
$$

Lemma 2.4 ([10]) Let $c>0, \gamma>0$. For any $x, y \in[0, c]$, we have that

(1) if $\gamma>1$, then $\left|x^{\gamma}-y^{\gamma}\right| \leq \gamma c^{\gamma-1}|x-y|$;

(2) if $0<\gamma \leq 1$, then $\left|x^{\gamma}-y^{\gamma}\right| \leq|x-y|^{\gamma}$.

\section{Existence result}

Denote by $C([0,1])$ the Banach space of all continuous functions from $[0,1]$ into $\mathbb{R}$ with the norm

$$
\|u\|=\max \{|u(t)|: t \in[0,1]\} .
$$

Define the cone $P$ in $C\left([0,1]^{2}\right)$ as $P=\{u \in C([0,1]): u(t) \geq 0, t \in[0,1]\}$. Let $q>1$ and $\tilde{q}>1$ satisfy the relation $\frac{1}{p}+\frac{1}{q}=1, \frac{1}{\tilde{p}}+\frac{1}{\tilde{q}}=1$, where $p, \tilde{p}$ are given by (1.4).

To prove the existence of solutions to (1.4), we need the following auxiliary lemma.

Lemma 3.1 Given $h_{1}, h_{2} \in C[0,1], \eta \in(0,1), \gamma \in\left(0, \frac{1}{\eta^{\alpha-\beta-1}}\right)$, and $\alpha \geq \beta+1$, the unique solution of $C$ boundary value problem for a coupled system

$$
\begin{aligned}
& \left(\varphi_{p}\left(D_{0^{+}}^{\alpha} u(t)\right)\right)^{\prime}+h_{1}(t)=0, \quad 0<t<1, \\
& \left(\varphi_{\tilde{p}}\left(D_{0^{+}}^{\alpha} v(t)\right)\right)^{\prime}+h_{2}(t)=0, \quad 0<t<1, \\
& D_{0^{+}}^{\alpha} u(0)=u(0)=u^{\prime}(0)=0, \quad D_{0^{+}}^{\beta} u(1)=\gamma D_{0^{+}}^{\beta} u(\eta), \\
& D_{0^{+}}^{\alpha} v(0)=v(0)=v^{\prime}(0)=0, \quad D_{0^{+}}^{\beta} v(1)=\gamma D_{0^{+}}^{\beta} v(\eta),
\end{aligned}
$$

is

$$
\begin{aligned}
u(t)= & \int_{0}^{1} G_{1}(t, s) \varphi_{q}\left(\int_{0}^{s} h_{1}(\tau) d \tau\right) d s \\
& +\frac{\gamma t^{\alpha-1}}{1-\gamma \eta^{\alpha-\beta-1}} \int_{0}^{1} G_{2}(\eta, s) \varphi_{q}\left(\int_{0}^{s} h_{1}(\tau) d \tau\right) d s
\end{aligned}
$$


and

$$
\begin{aligned}
v(t)= & \int_{0}^{1} G_{1}(t, s) \varphi_{\tilde{q}}\left(\int_{0}^{s} h_{2}(\tau) d \tau\right) d s \\
& +\frac{\gamma t^{\alpha-1}}{1-\gamma \eta^{\alpha-\beta-1}} \int_{0}^{1} G_{2}(\eta, s) \varphi_{\tilde{q}}\left(\int_{0}^{s} h_{2}(\tau) d \tau\right) d s,
\end{aligned}
$$

where

$$
\begin{aligned}
& G_{1}(t, s)= \begin{cases}\frac{t^{\alpha-1}(1-s)^{\alpha-\beta-1}-(t-s)^{\alpha-1}}{\Gamma(\alpha)}, & 0 \leq s \leq t \leq 1, \\
\frac{t^{\alpha-1}(1-s)^{\alpha-\beta-1}}{\Gamma(\alpha)}, & 0 \leq t \leq s \leq 1,\end{cases} \\
& G_{2}(\eta, s)= \begin{cases}\frac{[\eta(1-s)]^{\alpha-\beta-1}-(\eta-s)^{\alpha-\beta-1}}{\Gamma(\alpha)}, & 0 \leq s \leq \eta \leq 1, \\
\frac{[\eta(1-s)]^{\alpha-\beta-1}}{\Gamma(\alpha)}, & 0 \leq \eta \leq s \leq 1 .\end{cases}
\end{aligned}
$$

Proof Integrating equation (3.1) from 0 to $t$, we have

$$
\varphi_{p}\left(D_{0^{+}}^{\alpha} u(t)\right)-\varphi_{p}\left(D_{0^{+}}^{\alpha} u(0)\right)=\int_{0}^{t} h_{1}(s) d s
$$

and so,

$$
D_{0^{+}}^{\alpha} u(t)=-\varphi_{q}\left(\int_{0}^{t} h_{1}(s) d s\right) .
$$

From Lemma 2.2,

$$
\begin{aligned}
u(t) & =-I_{0^{+}}^{\alpha} \varphi_{q}\left(\int_{0}^{t} h_{1}(s) d s\right)+A t^{\alpha-1}+B t^{\alpha-2}+C t^{\alpha-3} \\
& =-\frac{1}{\Gamma(\alpha)} \int_{0}^{t}(t-s)^{\alpha-1} \varphi_{q}\left(\int_{0}^{s} h_{1}(\tau) d \tau\right) d s+A t^{\alpha-1}+B t^{\alpha-2}+C t^{\alpha-3} .
\end{aligned}
$$

From (3.3), $B=C=0$, and so

$$
u(t)=-I_{0^{+}}^{\alpha} \varphi_{q}\left(\int_{0}^{t} h_{1}(s) d s\right)+A t^{\alpha-1} .
$$

Now, from Remark 2.3

$$
\begin{aligned}
D_{0^{+}}^{\beta} u(t) & =-I_{0^{+}}^{\alpha-\beta} \varphi_{q}\left(\int_{0}^{t} h_{1}(s) d s\right)+A \frac{\Gamma(\alpha)}{\Gamma(\alpha-\beta)} t^{\alpha-\beta-1} \\
& =-\frac{1}{\Gamma(\alpha-\beta)} \int_{0}^{t}(t-s)^{\alpha-\beta-1} \varphi_{q}\left(\int_{0}^{s} h_{1}(\tau) d \tau\right) d s+A \frac{\Gamma(\alpha)}{\Gamma(\alpha-\beta)} t^{\alpha-\beta-1} .
\end{aligned}
$$

Therefore

$$
\begin{aligned}
& D_{0^{+}}^{\beta} u(1)=-\frac{1}{\Gamma(\alpha-\beta)} \int_{0}^{1}(1-s)^{\alpha-\beta-1} \varphi_{q}\left(\int_{0}^{s} h_{1}(\tau) d \tau\right) d s+A \frac{\Gamma(\alpha)}{\Gamma(\alpha-\beta)}, \\
& \gamma D_{0^{+}}^{\beta} u(\eta)=-\frac{\gamma}{\Gamma(\alpha-\beta)} \int_{0}^{\eta}(\eta-s)^{\alpha-\beta-1} \varphi_{q}\left(\int_{0}^{s} h_{1}(\tau) d \tau\right) d s+A \frac{\Gamma(\alpha) \gamma}{\Gamma(\alpha-\beta)} t^{\alpha-\beta-1} \eta^{\alpha-\beta-1} .
\end{aligned}
$$


By boundary condition (3.3), we have

$$
\begin{aligned}
A= & \frac{1}{1-\gamma \eta^{\alpha-\beta-1}} \int_{0}^{1} \frac{(1-s)^{\alpha-\beta-1}}{\Gamma(\alpha)} \varphi_{q}\left(\int_{0}^{s} h_{1}(\tau) d \tau\right) d s \\
& -\frac{\gamma}{1-\gamma \eta^{\alpha-\beta-1}} \int_{0}^{\eta} \frac{(\eta-s)^{\alpha-\beta-1}}{\Gamma(\alpha)} \varphi_{q}\left(\int_{0}^{s} h_{1}(\tau) d \tau\right) d s,
\end{aligned}
$$

and replacing in (3.7), we obtain

$$
\begin{aligned}
u(t)= & -\frac{1}{\Gamma(\alpha)} \int_{0}^{t}(t-s)^{\alpha-1} \varphi_{q}\left(\int_{0}^{s} h_{1}(\tau) d \tau\right) d s \\
& +\frac{t^{\alpha-1}}{1-\gamma \eta^{\alpha-\beta-1}} \int_{0}^{1} \frac{(1-s)^{\alpha-\beta-1}}{\Gamma(\alpha)} \varphi_{q}\left(\int_{0}^{s} h_{1}(\tau) d \tau\right) d s \\
& +\frac{\gamma t^{\alpha-1}}{1-\gamma \eta^{\alpha-\beta-1}} \int_{0}^{1} \frac{(\eta-s)^{\alpha-\beta-1}}{\Gamma(\alpha)} \varphi_{q}\left(\int_{0}^{s} h_{1}(\tau) d \tau\right) d s .
\end{aligned}
$$

Splitting the second integral in two parts of the form

$$
t^{\alpha-1}+\frac{k}{1-\gamma \eta^{\alpha-\beta-1}}=\frac{t^{\alpha-1}}{1-\gamma \eta^{\alpha-\beta-1}},
$$

we have $k=\gamma \eta^{\alpha-\beta-1} t^{\alpha-1}$, and thus

$$
\begin{aligned}
& u(t)=-\frac{1}{\Gamma(\alpha)} \int_{0}^{t}(t-s)^{\alpha-1} \varphi_{q}\left(\int_{0}^{s} h_{1}(\tau) d \tau\right) d s \\
& +t^{\alpha-1} \int_{0}^{1} \frac{(1-s)^{\alpha-\beta-1}}{\Gamma(\alpha)} \varphi_{q}\left(\int_{0}^{s} h_{1}(\tau) d \tau\right) d s \\
& +\frac{\gamma \eta^{\alpha-\beta-1} t^{\alpha-1}}{1-\gamma \eta^{\alpha-\beta-1}} \int_{0}^{1} \frac{(1-s)^{\alpha-\beta-1}}{\Gamma(\alpha)} \varphi_{q}\left(\int_{0}^{s} h_{1}(\tau) d \tau\right) d s \\
& -\frac{\gamma t^{\alpha-1}}{1-\gamma \eta^{\alpha-\beta-1}} \int_{0}^{\eta} \frac{(\eta-s)^{\alpha-\beta-1}}{\Gamma(\alpha)} \varphi_{q}\left(\int_{0}^{s} h_{1}(\tau) d \tau\right) d s \\
& =\int_{0}^{t} \frac{t^{\alpha-1}(1-s)^{\alpha-\beta-1}-(t-s)^{\alpha-1}}{\Gamma(\alpha)} \varphi_{q}\left(\int_{0}^{s} h_{1}(\tau) d \tau\right) d s \\
& +\int_{t}^{1} \frac{t^{\alpha-1}(1-s)^{\alpha-\beta-1}}{\Gamma(\alpha)} \varphi_{q}\left(\int_{0}^{s} h_{1}(\tau) d \tau\right) d s \\
& +\frac{\gamma t^{\alpha-1}}{1-\gamma \eta^{\alpha-\beta-1}} \int_{0}^{\eta} \frac{[\eta(1-s)]^{\alpha-\beta-1}-(\eta-s)^{\alpha-\beta-1}}{\Gamma(\alpha)} \times \varphi_{q}\left(\int_{0}^{s} h_{1}(\tau) d \tau\right) d s \\
& +\frac{\gamma t^{\alpha-1}}{1-\gamma \eta^{\alpha-\beta-1}} \int_{0}^{\eta} \frac{[\eta(1-s)]^{\alpha-\beta-1}}{\Gamma(\alpha)} \times \varphi_{q}\left(\int_{0}^{s} h_{1}(\tau) d \tau\right) d s \\
& =\int_{0}^{1} G_{1}(t, s) \varphi_{q}\left(\int_{0}^{s} h_{1}(\tau) d \tau\right) d s+\frac{\gamma t^{\alpha-1}}{1-\gamma \eta^{\alpha-\beta-1}} \\
& \times \int_{0}^{1} G_{2}(\eta, s) \varphi_{q}\left(\int_{0}^{s} h_{1}(\tau) d \tau\right) d s
\end{aligned}
$$

This completes the proof. 
Lemma 3.2 ([35]) Let $\rho \in(0,1)$ be fixed. The kernel $G_{1}(t, s)$ satisfies the following properties:

(1) $G_{1}(t, s) \in C([0,1] \times[0,1])$ and $G_{1}(t, s)>0$ for all $s, t \in(0,1)$;

(2) $G_{1}(t, s) \leq G_{1}(1, s)$ for all $s \in(0,1)$;

(3) $\min _{\rho \leq t \leq 1} G_{1}(t, s) \geq \rho^{\alpha-1} G_{1}(1, s)$ for all $s \in[0,1]$.

We are now ready to present our main result. In this section we give an existence result based on the nonlinear alternative of Leray-Schauder type.

Theorem 3.1 Assume $\left(H_{1}\right)-\left(H_{2}\right)$ and that the following condition holds:

$\left(H_{3}\right)$ There exist functions $p, q, h, \breve{p}, \breve{q}$, and $\bar{h} \in L^{1}\left([0,1], \mathbb{R}_{+}\right)$and constants $\alpha_{1}, \alpha_{2}, \alpha_{3}$, and $\alpha_{4} \in[0,1)$ such that

$$
|f(u, v)| \leq p(t)|u|^{\alpha_{1}}+q(t)|v|^{\alpha_{2}}+h(t) \quad \text { for each } t \in[0,1] \text { and } u, v \in \mathbb{R}
$$

and

$$
|g(u, v)| \leq \breve{p}(t)|u|^{\alpha_{3}}+\breve{q}(t)|v|^{\alpha_{4}}+\breve{h}(t) \quad \text { for each } t \in[0,1] \text { and } u, v \in \mathbb{R} \text {. }
$$

If $\alpha_{1} p, \alpha_{2} p, \alpha_{3} q$, and $\alpha_{4} q \in[0,1)$, then system (1.4) has at least one solution.

Proof Let $N$ be the operator

$$
N: C(0,1) \times C(0,1) \rightarrow C(0,1) \times C(0,1)
$$

defined by

$$
N(u, v)=\left(N_{1}(u, v), N_{2}(u, v)\right),
$$

where

$$
\begin{aligned}
& N_{1}(u, v)(t) \\
& =\int_{0}^{1} G_{1}(t, s) \varphi_{q}\left(\int_{0}^{s} a_{1}(\tau) f\left(u\left(\theta_{1}(\tau)\right), v\left(\theta_{2}(\tau)\right)\right) d \tau\right) d s \\
& \quad+\frac{\gamma t^{\alpha-1}}{1-\gamma \eta^{\alpha-\beta-1}} \int_{0}^{1} G_{2}(\eta, s) \varphi_{q}\left(\int_{0}^{s} a_{1}(\tau) f\left(u\left(\theta_{1}(\tau)\right), v\left(\theta_{2}(\tau)\right)\right) d \tau\right) d s,
\end{aligned}
$$

and

$$
\begin{aligned}
N_{2}(u, v)= & \int_{0}^{1} G_{1}(t, s) \varphi_{\tilde{q}}\left(\int_{0}^{s} a_{2}(\tau) g\left(u\left(\theta_{1}(\tau)\right), v\left(\theta_{2}(\tau)\right)\right) d \tau\right) d s \\
& +\frac{\gamma t^{\alpha-1}}{1-\gamma \eta^{\alpha-\beta-1}} \int_{0}^{1} G_{2}(\eta, s) \varphi_{\tilde{q}}\left(\int_{0}^{s} a_{2}(\tau) g\left(u\left(\theta_{1}(\tau)\right), v\left(\theta_{2}(\tau)\right)\right) d \tau\right) d s
\end{aligned}
$$

We shall use the Leray-Schauder fixed point theorem to prove that $N$ has a fixed point. The proof will be given in several steps. 
Step 1 . To show that $N$ is continuous, let $\left(u_{n}, v_{n}\right)$ be a sequence such that $\left(u_{n}, v_{n}\right) \rightarrow$ $(u, v) \in C[0,1] \times C[0,1]$ as $n \rightarrow \infty$. Then we have

$$
\begin{aligned}
& \left|N_{1}\left(u_{n}, v_{n}\right)(t)-N_{1}(u, v)(t)\right| \\
& =\mid \int_{0}^{1} G_{1}(t, s) \varphi_{q}\left(\int_{0}^{s} a_{1}(\tau) f\left(u_{n}\left(\theta_{1}(\tau)\right), v_{n}\left(\theta_{2}(\tau)\right)\right) d \tau\right) d s \\
& \quad+\frac{\gamma t^{\alpha-1}}{1-\gamma \eta^{\alpha-\beta-1}} \int_{0}^{1} G_{2}(\eta, s) \varphi_{q}\left(\int_{0}^{s} a_{1}(\tau) f\left(u_{n}\left(\theta_{1}(\tau)\right), v_{n}\left(\theta_{2}(\tau)\right)\right) d \tau\right) d s \\
& \quad-\left[\int_{0}^{1} G_{1}(t, s) \varphi_{q}\left(\int_{0}^{s} a_{1}(\tau) f\left(u\left(\theta_{1}(\tau)\right), v\left(\theta_{2}(\tau)\right)\right) d \tau\right) d s\right. \\
& \left.\quad+\int_{0}^{1} \frac{\gamma t^{\alpha-1} G_{2}(\eta, s)}{1-\gamma \eta^{\alpha-\beta-1}} \varphi_{q}\left(\int_{0}^{s} a_{1}(\tau) f\left(u\left(\theta_{1}(\tau)\right), v\left(\theta_{2}(\tau)\right)\right) d \tau\right) d s\right] .
\end{aligned}
$$

By Lemma 3.2 and $t \in[0,1]$,

$$
\begin{aligned}
& \left|N_{1}\left(u_{n}, v_{n}\right)(t)-N_{1}(u, v)(t)\right| \\
& \leq \int_{0}^{1} G_{1}(1, s)\left(\int_{0}^{s}\left|a_{1}(\tau) f\left(u_{n}\left(\theta_{1}(\tau)\right), v_{n}\left(\theta_{2}(\tau)\right)\right)\right|^{q-1}\right. \\
& \left.\quad-\left|a_{1}(\tau) f\left(u\left(\theta_{1}(\tau)\right), v\left(\theta_{2}(\tau)\right)\right)\right|^{q-1} d \tau\right) d s \\
& \quad+\frac{\gamma}{1-\gamma \eta^{\alpha-\beta-1}} \int_{0}^{1} G_{2}(\eta, s)\left(\int_{0}^{s}\left|a_{1}(\tau) f\left(u_{n}\left(\theta_{1}(\tau)\right), v_{n}\left(\theta_{2}(\tau)\right)\right) d \tau\right|^{q-1}\right. \\
& \left.\quad-\left|a_{1}(\tau) f\left(u\left(\theta_{1}(\tau)\right), v\left(\theta_{2}(\tau)\right)\right)\right|^{q-1} d \tau\right) d s .
\end{aligned}
$$

On the other hand, since $f$ is a continuous function combined with the fact that

$$
\left\|u_{n}-u\right\| \rightarrow 0, \quad \text { as } n \rightarrow \infty
$$

then there exists $N \geq 1$ such that for all $\tau \in[0,1]$ the following estimate

$$
\left.\mid f\left(u_{n}\left(\theta_{1}(\tau)\right), v_{n}\left(\theta_{2}(\tau)\right)\right) d \tau\right)-f\left(u\left(\theta_{1}(\tau)\right), v\left(\theta_{2}(\tau)\right)\right) \mid<\epsilon,
$$

holds for $n \geq N$. By the Lebesgue dominated convergence theorem, we have

$$
\left\|N_{1}\left(u_{n}, v_{n}\right)-N_{1}(u, v)\right\| \rightarrow 0, \quad \text { as } n \rightarrow \infty .
$$

Similarly,

$$
\left\|N_{2}\left(u_{n}, v_{n}\right)-N_{2}(u, v)\right\| \rightarrow 0, \quad \text { as } n \rightarrow \infty .
$$

Consequently, $N$ is continuous.

Step 2. $N$ maps bounded sets into bounded sets in $C[0,1] \times C[0,1]$, it suffices to show that for any $r>0$ there exists a positive constant vector $l=\left(l_{1}, l_{2}\right)$ such that, for each $(u, v) \in$ 
$B_{r}=\{(u, v) \in C[0,1] \times C[0,1]:\|u\| \leq r,\|v\| \leq r\}$, we have

$$
\|N(u, v)\| \leq l .
$$

For each $t \in[0,1]$, we have

$$
\begin{aligned}
\left|N_{1}(u, v)(t)\right| & \int_{0}^{1}\left|G_{1}(t, s)\right| \varphi_{q}\left(\int_{0}^{s}\left|a_{1}(\tau) f\left(u\left(\theta_{1}(\tau)\right), v\left(\theta_{2}(\tau)\right)\right) d \tau\right|\right) d s \\
& +\frac{\gamma t^{\alpha-1}}{1-\gamma \eta^{\alpha-\beta-1}} \int_{0}^{1}\left|G_{2}(\eta, s)\right| \varphi_{q}\left(\int_{0}^{s}\left|a_{1}(\tau) f\left(u\left(\theta_{1}(\tau)\right), v\left(\theta_{2}(\tau)\right)\right) d \tau\right|\right) d s \\
\leq & \max \left\{2^{q-1}, 1\right\} \int_{0}^{1} G_{1}(1, s) \int_{0}^{s}\left|a_{1}(\tau)\right|^{q-1}|p(\tau)|^{q-1}\left|u\left(\theta_{1}(\tau)\right)\right|^{\alpha_{1}(q-1)} \\
& +\left|a_{1}(\tau)\right|^{q-1}|q(\tau)|^{q-1}\left|v\left(\theta_{2}(\tau)\right)\right|^{\alpha_{2}(q-1)}+\left|a_{1}(\tau)\right|^{q-1}|h(\tau)|^{q-1} d \tau d s \\
& +\max \left\{2^{q-1}, 1\right\} \frac{\gamma t^{\alpha-1}}{1-\gamma \eta^{\alpha-\beta-1}} \int_{0}^{1}\left|G_{2}(\eta, s)\right| \int_{0}^{s}\left|a_{1}(\tau)\right|^{q-1}|p(\tau)|^{q-1}\left|u\left(\theta_{1}(\tau)\right)\right|^{\alpha_{1}(q-1)} \\
& +\left|a_{1}(\tau)\right|^{q-1}|q(\tau)|^{q-1}\left|v\left(\theta_{2}(\tau)\right)\right|^{\alpha_{2}(q-1)}+\left|a_{1}(\tau)\right|^{q-1}|h(\tau)|^{q-1} d \tau d s \\
\leq & \max \left\{2^{q-1}, 1\right\}\left(\|u\|^{\alpha_{1}(q-1)}\left\|a_{1}\right\|_{L_{1}}^{q-1}\|p\|_{L_{1}}^{q-1}+\|v\|^{\alpha_{2}(q-1)}\left\|a_{1}\right\|_{L_{1}}^{q-1}\|q\|_{L_{1}}^{q-1}\right. \\
& \left.+\left\|a_{1}\right\|_{L_{1}}^{q-1}\|h\|_{L_{1}}^{q-1}\right) \int_{0}^{1} \frac{(1-s)^{\alpha-\beta-1}}{\Gamma(\alpha)}+\frac{\gamma t^{\alpha-1}}{1-\gamma \eta^{\alpha-\beta-1}}\left(\|u\|^{\alpha_{1}(q-1)}\left\|a_{1}\right\|_{L_{1}}^{q-1}\|p\|_{L_{1}}^{q-1}\right. \\
& \left.+\|v\|^{\alpha_{2}(q-1)}\left\|a_{1}\right\|_{L_{1}}^{q-1}\|q\|_{L_{1}}^{q-1}+\left\|a_{1}\right\|_{L_{1}}^{q-1}\|h\|_{L_{1}}^{q-1}\right) \int_{0}^{1} \frac{\eta^{\alpha-\beta-1}(1-s)^{\alpha-\beta-1}}{\Gamma(\alpha)} \\
\leq & \frac{\max \left\{2^{q-1}, 1\right\}}{(\alpha-\beta) \Gamma(\alpha)}\left(r^{\alpha_{1}(q-1)}\left\|a_{1}\right\|_{L_{1}}^{q-1}\|p\|_{L_{1}}^{q-1}+r^{\alpha_{2}(q-1)}\left\|a_{1}\right\|_{L_{1}}^{q-1}\|q\|_{L_{1}}^{q-1}+\left\|a_{1}\right\|_{L_{1}}^{q-1}\|h\|_{L_{1}}^{q-1}\right) \\
& +\frac{\max \left\{2^{q-1}, 1\right\} \eta^{\alpha-\beta-1} \gamma}{\left(1-\gamma \eta^{\alpha-\beta-1}\right)(\alpha-\beta) \Gamma(\alpha)}\left(r^{\alpha_{1}(q-1)}\left\|a_{1}\right\|_{L_{1}}^{q-1}\|p\|_{L_{1}}^{q-1}+r^{\alpha_{2}(q-1)}\left\|a_{1}\right\|_{L_{1}}^{q-1}\|q\|_{L_{1}}^{q-1}\right. \\
& \left.+\left\|a_{1}\right\|_{L_{1}}^{q-1}\|h\|_{L_{1}}^{q-1}\right) .
\end{aligned}
$$

Hence

$$
\begin{aligned}
& \left\|N_{1}(u, v)\right\| \\
& \leq \frac{\max \left\{2^{q-1}, 1\right\}}{(\alpha-\beta) \Gamma(\alpha)}\left(r^{\alpha_{1}(q-1)}\left\|a_{1}\right\|_{L_{1}}^{q-1}\|p\|_{L_{1}}^{q-1}+r^{\alpha_{2}(q-1)}\left\|a_{1}\right\|_{L_{1}}^{q-1}\|q\|_{L_{1}}^{q-1}+\left\|a_{1}\right\|_{L_{1}}^{q-1}\|h\|_{L_{1}}^{q-1}\right) \\
& \quad+\frac{\max \left\{2^{q-1}, 1\right\} \eta^{\alpha-\beta-1} \gamma}{\left(1-\gamma \eta^{\alpha-\beta-1}\right)(\alpha-\beta) \Gamma(\alpha)}\left(r^{\alpha_{1}(q-1)}\left\|a_{1}\right\|_{L_{1}}^{q-1}\|p\|_{L_{1}}^{q-1}+r^{\alpha_{2}(q-1)}\left\|a_{1}\right\|_{L_{1}}^{q-1}\|q\|_{L_{1}}^{q-1}\right. \\
& \left.\quad+\left\|a_{1}\right\|_{L_{1}}^{q-1}\|h\|_{L_{1}}^{q-1}\right):=l_{1} .
\end{aligned}
$$

Similarly, we have

$$
\begin{aligned}
& \left\|N_{2}(u, v)\right\| \\
& \quad \leq \frac{\max \left\{2^{\tilde{q}-1}, 1\right\}}{(\alpha-\beta) \Gamma(\alpha)}\left(r^{\alpha_{3}(\tilde{q}-1)}\left\|a_{2}\right\|_{L_{1}}^{\tilde{q}-1}\|\breve{p}\|_{L_{1}}^{\tilde{q}-1}+r^{\alpha_{4}(\tilde{q}-1)}\left\|a_{2}\right\|_{L_{1}}^{\tilde{q}-1}\|\breve{q}\|_{L_{1}}^{\tilde{q}-1}+\left\|a_{2}\right\|_{L_{1}}^{\tilde{q}-1}\|\breve{h}\|_{L_{1}}^{\tilde{q}-1}\right)
\end{aligned}
$$




$$
\begin{aligned}
& +\frac{\max \left\{2^{\tilde{q}-1}, 1\right\} \eta^{\alpha-\beta-1} \gamma}{\left(1-\gamma \eta^{\alpha-\beta-1}\right)(\alpha-\beta) \Gamma(\alpha)}\left(r^{\alpha_{3}(\tilde{q}-1)}\left\|a_{2}\right\|_{L_{1}}^{\tilde{q}-1}\|\breve{p}\|_{L_{1}}^{\tilde{q}-1}+r^{\alpha_{4}(\tilde{q}-1)}\left\|a_{2}\right\|_{L_{1}}^{\tilde{q}-1}\|\breve{q}\|_{L_{1}}^{\tilde{q}-1}\right. \\
& \left.+\left\|a_{2}\right\|_{L_{1}}^{\tilde{q}-1}\|\breve{h}\|_{L_{1}}^{\tilde{q}-1}\right):=l_{2} .
\end{aligned}
$$

Step 3. $N$ maps bounded sets into equicontinuous. Let $u \in B_{r}$ be a bounded set as in Step $2, t_{1}, t_{2} \in[0,1]$ with $t_{1}<t_{2}$, from (3.5) and Lemma 2.3, we have

$$
\begin{aligned}
\left|N_{1}(u, v)\left(t_{2}\right)-N_{1}(u, v)\left(t_{1}\right)\right| & \\
\leq & \int_{0}^{1}\left|G_{1}\left(t_{2}, s\right)-G_{1}\left(t_{1}, s\right)\right| \varphi_{q}\left(\int_{0}^{s} \mid a_{1}(\tau) f\left(u\left(\theta_{1}(\tau)\right), v\left(\theta_{2}(\tau)\right)\right) d \tau\right) \mid d s \\
& +\frac{\gamma\left|t_{2}-t_{1}\right|^{\alpha-1}}{1-\gamma \eta^{\alpha-\beta-1}} \int_{0}^{1}\left|G_{2}(\eta, s)\right| \\
& \times \varphi_{q}\left(\int_{0}^{s}\left|a_{1}\right|(\tau) f\left(u\left(\theta_{1}(\tau)\right), v\left(\theta_{2}(\tau)\right)\right) d \tau\right) \mid d s \\
\leq & \int_{0}^{1}\left|G_{1}\left(t_{2}, s\right)-G_{1}\left(t_{1}, s\right)\right| \int_{0}^{s} \mid a_{1}(\tau)\left[p(\tau) \mid u\left(\left.\theta_{1}\right|^{\alpha_{1}}+q(\tau)\left|v\left(\theta_{2}(\tau)\right)\right|^{\alpha_{2}}\right.\right. \\
& +h(\tau) d \tau d s]\left.\right|^{q-1}+\frac{\gamma\left|t_{2}-t_{1}\right|^{\alpha-1}}{1-\gamma \eta^{\alpha-\beta-1}} \int_{0}^{1}\left|G_{2}(\eta, s)\right| \\
& \times \int_{0}^{s} \mid a_{1}(\tau)\left[p(\tau)\left|u\left(\left.\theta_{1}\right|^{\alpha_{1}}+q(\tau)\left|v\left(\theta_{2}(\tau)\right)\right|^{\alpha_{2}}+h(\tau) d \tau d s\right]\right|^{q-1}\right. \\
\leq & \max \left\{2^{q-1}, 1\right\} \int_{0}^{1}\left|G_{1}\left(t_{2}, s\right)-G_{1}\left(t_{1}, s\right)\right| \int_{0}^{s}\left|a_{1}(\tau)\right|^{q-1}|p(\tau)|^{q-1} \\
& \times\left|u\left(\theta_{1}(\tau)\right)\right|^{\alpha_{1}(q-1)}+\left|a_{1}(\tau)\right|^{q-1}|q(\tau)|^{q-1}\left|v\left(\theta_{2}(\tau)\right)\right|^{\alpha_{2}(q-1)}+\left|a_{1}(\tau)\right|^{q-1} \\
& \times|h(\tau)|^{q-1} d \tau d s+\max \left\{2^{q-1}, 1\right\} \frac{\gamma\left|t_{2}-t_{1}\right|^{\alpha-1}}{1-\gamma \eta^{\alpha-\beta-1}} \int_{0}^{1}\left|G_{2}(\eta, s)\right| \\
& \times\left.\int_{0}^{s}\left|a_{1}(\tau)\right|^{q-1}|p(\tau)|^{q-1} u\left(\theta_{1}(\tau)\right)\right|^{\alpha_{1}(q-1)} \\
& +\left|a_{1}(\tau)\right|^{q-1}|q(\tau)|^{q-1}\left|v\left(\theta_{2}(\tau)\right)\right|^{\alpha_{2}(q-1)}+\left|a_{1}(\tau)\right|^{q-1}|h(\tau)|^{q-1} d \tau d s .
\end{aligned}
$$

By Lemma 2.4 we obtain

$$
\begin{aligned}
\leq & \max \left\{2^{q-1}, 1\right\}\left(r^{\alpha_{1}(q-1)}\left\|a_{1}\right\|_{L_{1}}^{q-1}\|p\|_{L_{1}}^{q-1}+r^{\alpha_{2}(q-1)}\left\|a_{1}\right\|_{L_{1}}^{q-1}\|q\|_{L_{1}}^{q-1}\right. \\
& \left.+\left\|a_{1}\right\|_{L_{1}}^{q-1}\|h\|_{L_{1}}^{q-1}\right) \int_{0}^{1}\left|G_{1}\left(t_{1}, s\right)-G_{1}\left(t_{2}, s\right)\right| d s \\
& +\frac{\max \left\{2^{q-1}, 1\right\} \gamma(\alpha-1)\left|t_{2}-t_{1}\right|}{\left(1-\gamma \eta^{\alpha-\beta-1}\right)}\left(r^{\alpha_{1}(q-1)}\left\|a_{1}\right\|_{L_{1}}^{q-1}\|p\|_{L_{1}}^{q-1}\right. \\
& \left.+r^{\alpha_{2}(q-1)}\left\|a_{1}\right\|_{L_{1}}^{q-1}\|q\|_{L_{1}}^{q-1}+\left\|a_{1}\right\|_{L_{1}}^{q-1}\|h\|_{L_{1}}^{q-1}\right) \int_{0}^{1}\left|G_{2}(\eta, s)\right| d s .
\end{aligned}
$$

Similarly, we have

$$
\begin{aligned}
& \left|N_{2}(u, v)\left(t_{1}\right)-N_{2}(u, v)\left(t_{2}\right)\right| \\
& \quad \leq \max \left\{2^{\tilde{q}-1}, 1\right\}\left(r^{\alpha_{3}(q-1)}\left\|a_{2}\right\|_{L_{1}}^{\tilde{q}-1}\|\breve{p}\|_{L_{1}}^{\tilde{q}-1}+r^{\alpha_{4}(\tilde{q}-1)}\left\|a_{2}\right\|_{L_{1}}^{\tilde{q}-1}\|\breve{q}\|_{L_{1}}^{\tilde{q}-1}\right.
\end{aligned}
$$




$$
\begin{aligned}
& \left.+\left\|a_{2}\right\|_{L_{1}}^{\tilde{q}-1}\|\breve{h}\|_{L_{1}}^{\tilde{q}-1}\right) \int_{0}^{1}\left|G_{1}\left(t_{1}, s\right)-G_{1}\left(t_{2}, s\right)\right| d s \\
& +\frac{\max \left\{2^{\tilde{q}-1}, 1\right\} \gamma(\alpha-1)\left|t_{2}-t_{1}\right|}{\left(1-\gamma \eta^{\alpha-\beta-1}\right)}\left(r^{\alpha_{3}(\tilde{q}-1)}\left\|a_{2}\right\|_{L_{1}}^{\tilde{q}-1}\|\breve{p}\|_{L_{1}}^{\tilde{q}-1}\right. \\
& \left.+r^{\alpha_{4}(\tilde{q}-1)}\left\|a_{2}\right\|_{L_{1}}^{\tilde{q}-1}\|\breve{q}\|_{L_{1}}^{\tilde{q}-1}+\left\|a_{2}\right\|_{L_{1}}^{\tilde{q}-1}\|\breve{h}\|_{L_{1}}^{\tilde{q}-1}\right) \int_{0}^{1}\left|G_{2}(\eta, s)\right| d s .
\end{aligned}
$$

The continuity of $G_{1}$ implies that the right-hand side of the above inequality tends to zero if $t_{2} \rightarrow t_{1}$. Therefore, by Arzela-Ascoli $N$ is completely continuous.

Step 4. A priori bounds. Now it remains to show that the set

$$
\mathcal{M}=\{(u, v) C([0,1], \mathbb{R}) \times C([0,1], \mathbb{R}):(u, v)=\lambda N(u, v)<\lambda<1\}
$$

is bounded. Let $(u, v) \in \mathcal{M}$, then there exists $0<\lambda<1$ such that $u=\lambda N_{1}(u, v)$ and $v=$ $\lambda N_{2}(u, v)$. Thus, for $t \in[0,1]$, we have

$$
\begin{aligned}
|u(t)| \leq & \int_{0}^{1}\left|G_{1}(t, s)\right| \varphi_{q}\left(\int_{0}^{s} \mid a_{1}(\tau) f\left(u\left(\theta_{1}(\tau)\right), v\left(\theta_{2}(\tau)\right)\right) d \tau\right) \mid d s \\
& +\frac{\gamma t^{\alpha-1}}{1-\gamma \eta^{\alpha-\beta-1}} \int_{0}^{1}\left|G_{2}(\eta, s)\right| \varphi_{q}\left(\int_{0}^{s} \mid a_{1}(\tau) f\left(u\left(\theta_{1}(\tau)\right), v\left(\theta_{2}(\tau)\right)\right) d \tau\right) \mid d s \\
\leq & \max \left\{2^{q-1}, 1\right\}\left[\|p\|_{L^{1}}^{q-1}\left\|a_{1}\right\|_{L^{1}}^{q-1}\|u\|^{\alpha_{1}(q-1)}+\|q\|_{L^{1}}^{q-1}\left\|a_{1}\right\|_{L^{1}}^{q-1}\|v\|^{\alpha_{2}(q-1)}\right. \\
& \left.+\left\|a_{1}\right\|_{L^{1}}^{q-1}\|h\|_{L^{1}}^{q-1}\right] \\
& \times \int_{0}^{1} G_{1}(1, s) d s+\frac{\gamma}{1-\gamma \eta^{\alpha-\beta-1}}\left[\|p\|_{L^{1}}^{q-1}\left\|a_{1}\right\|_{L^{1}}^{q-1}\|u\|^{\alpha_{1}(q-1)}\right. \\
& +\|q\|_{L^{1}}^{q-1}\left\|a_{1}\right\|_{L^{1}}^{q-1}\|v\|^{\alpha_{2}(q-1)} \\
& \left.+\left\|a_{1}\right\|_{L^{1}}^{q-1}\|h\|_{L^{1}}^{q-1}\right] \int_{0}^{1}\left|G_{2}(\eta, s)\right| d s, \\
\|u\| \leq & \max \left\{2^{q-1}, 1\right\}\left[\|p\|_{L^{1}}^{q-1}\left\|a_{1}\right\|_{L^{1}}^{q-1}\|u\|^{\alpha_{1}(q-1)}+\|q\|_{L^{1}}^{q-1}\left\|a_{1}\right\|_{L^{1}}^{q-1}\|v\|^{\alpha_{2}(q-1)}\right. \\
& \left.+\left\|a_{1}\right\|_{L^{1}}^{q-1}\|h\|_{L^{1}}^{q-1}\right]\left[\int_{0}^{1} \frac{(1-s)^{\alpha-\beta-1}}{\Gamma(\alpha)}+\frac{\gamma}{1-\gamma \eta^{\alpha-\beta-1}} \frac{\eta^{\alpha-\beta-1}(1-s)^{\alpha-\beta-1}}{\Gamma(\alpha)} d s\right], \\
\|u\| \leq & \max \left\{2^{q-1}, 1\right\}\left[\|p\|_{L^{1}}^{q-1}\left\|a_{1}\right\|_{L^{1}}^{q-1}\|u\|^{\alpha_{1}(q-1)}+\|q\|_{L^{1}}^{q-1}\left\|a_{1}\right\|_{L^{1}}^{q-1}\|v\|^{\alpha_{2}(q-1)}\right. \\
& \left.+\left\|a_{1}\right\|_{L^{1}}^{q-1}\|h\|_{L^{1}}^{q-1}\right] \frac{1}{\left(1-\gamma \eta^{\alpha-\beta-1}\right) \Gamma(\alpha)} \int_{0}^{1}(1-s)^{\alpha-\beta-1} d s .
\end{aligned}
$$

Hence,

$$
\begin{aligned}
\|u\| \leq & \frac{\max \left\{2^{q-1}, 1\right\}}{(\alpha-\beta)\left(1-\gamma \eta^{\alpha-\beta-1}\right) \Gamma(\alpha)}\left[\|p\|_{L^{1}}^{q-1}\left\|a_{1}\right\|_{L^{1}}^{q-1}\|u\|^{\alpha_{1}(q-1)}+\|q\|_{L^{1}}^{q-1}\left\|a_{1}\right\|_{L^{1}}^{q-1}\|v\|^{\alpha_{2}(q-1)}\right. \\
& \left.+\left\|a_{1}\right\|_{L^{1}}^{q-1}\|h\|_{L^{1}}^{q-1}\right] .
\end{aligned}
$$

Similarly, we obtain

$$
\|v\| \leq \frac{\max \left\{2^{q-1}, 1\right\}}{(\alpha-\beta)\left(1-\gamma \eta^{\alpha-\beta-1}\right) \Gamma(\alpha)}\left[\|\breve{p}\|_{L^{1}}^{\tilde{q}-1}\left\|a_{2}\right\|_{L^{1}}^{\tilde{q}-1}\|u\|^{\alpha_{3}(\tilde{q}-1)}+\|\breve{q}\|_{L^{1}}^{\tilde{q}-1}\left\|a_{2}\right\|_{L^{1}}^{\tilde{q}-1}\|v\|^{\alpha_{4}(\tilde{q}-1)}\right.
$$




$$
\left.+\left\|a_{2}\right\|_{L^{1}}^{\tilde{q}-1}\|\breve{h}\|_{L^{1}}^{\tilde{q}-1}\right]
$$

Notice that if $\epsilon \leq \delta$ and $\|u\|>1$, then $\|u\|^{\epsilon} \leq\|u\|^{\delta}$ Thus, $\|u\|^{\epsilon} \leq 1+\|u\|^{\delta}$ for all $u$. We then have

$$
\begin{aligned}
\|u\|+ & \|v\| \\
\leq & \frac{\max \left\{2^{q-1}, 1\right\}}{(\alpha-\beta)\left(1-\gamma \eta^{\alpha-\beta-1}\right) \Gamma(\alpha)}\left[\|p\|_{L^{1}}^{q-1}\left\|a_{1}\right\|_{L^{1}}^{q-1}\|u\|^{\alpha_{1}(q-1)}+\|q\|_{L^{1}}^{q-1}\left\|a_{1}\right\|_{L^{1}}^{q-1}\|v\|^{\alpha_{2}(q-1)}\right. \\
& \left.+\left\|a_{1}\right\|_{L^{1}}^{q-1}\|h\|_{L^{1}}^{q-1}\right]+\frac{\max \left\{2^{q-1}, 1\right\} \varphi_{q}\left(\int_{0}^{1} a_{1}(\tau) d \tau\right)}{(\alpha-\beta)\left(1-\gamma \eta^{\alpha-\beta-1}\right) \Gamma(\alpha)}\left[\|\breve{p}\|_{L^{1}}^{\tilde{q}-1}\left\|a_{2}\right\|_{L^{1}}^{\tilde{q}-1}\|u\|^{\alpha_{3}(\tilde{q}-1)}\right. \\
& \left.+\|\breve{q}\|_{L^{1}}^{\tilde{q}-1}\left\|a_{2}\right\|_{L^{1}}^{\tilde{q}-1}\|v\|^{\alpha_{4}(\tilde{q}-1)}+\left\|a_{2}\right\|_{L^{1}}^{\tilde{q}-1}\|\breve{h}\|_{L^{1}}^{\tilde{q}-1}\right] \\
\leq & \frac{\max \left\{2^{q-1}, 1\right\}}{(\alpha-\beta)\left(1-\gamma \eta^{\alpha-\beta-1}\right) \Gamma(\alpha)}\left(\|p\|_{L^{1}}^{q-1}\left\|a_{1}\right\|_{L^{1}}^{q-1}+\left\|a_{2}\right\|_{L^{1}}^{\tilde{q}-1}\|\breve{q}\|_{L^{1}}^{\tilde{q}-1}\right) \\
& \times\left(\|u\|^{\alpha_{1}(q-1)}+\|v\|^{\alpha_{4}(\tilde{q}-1)}\right) \\
& +\left(\left\|a_{2}\right\|_{L^{1}}^{\tilde{q}-1}\|\breve{p}\|_{L^{1}}^{\tilde{q}-1}+\left\|a_{1}\right\|_{L^{1}}^{q-1}\|q\|_{L^{1}}^{q-1}\right)\left(\|u\|^{\alpha_{3}(\tilde{q}-1)}+\|v\|^{\alpha_{2}(q-1)}\right) \\
& +\left(\left\|a_{1}\right\|_{L^{1}}^{q-1}\|h\|_{L^{1}}^{q-1}+\left\|a_{2}\right\|_{L^{1}}^{\tilde{q}-1}\|\breve{h}\|_{L^{1}}^{\tilde{q}-1}\right) \\
\leq & \frac{\max \left\{2^{q-1}, 1\right\}}{(\alpha-\beta)\left(1-\gamma \eta^{\alpha-\beta-1}\right) \Gamma(\alpha)}\left(\left\|a_{1}\right\|_{L^{1}}^{q-1}\|p\|_{L^{1}}^{q-1}+\left\|a_{2}\right\|_{L^{1}}^{\tilde{q}-1}\|\breve{q}\|_{L^{1}}^{\tilde{q}-1}+\left\|a_{2}\right\|_{L^{1}}^{\tilde{q}-1}\|\breve{p}\|_{L^{1}}^{\tilde{q}-1}\right. \\
& \left.+\left\|a_{1}\right\|_{L^{1}}^{q-1}\|q\|_{L^{1}}^{q-1}\right)\left(\|u\|^{\alpha_{\star}}+\|v\|^{\alpha_{\star}}\right)+\left(\left\|a_{1}\right\|_{L^{1}}^{q-1}\|h\|_{L^{1}}^{q-1}+\left\|a_{2}\right\|_{L^{1}}^{\tilde{q}-1}\|\breve{h}\|_{L^{1}}^{\tilde{q}-1}\right) \\
\leq & \frac{\max \left\{2^{q-1}, 1\right\}}{(\alpha-\beta)\left(1-\gamma \eta^{\alpha-\beta-1}\right) \Gamma(\alpha)} \\
& \times\left(\|p\|_{L^{1}}^{q-1}\left\|a_{1}\right\|_{L^{1}}^{q-1}+\|\breve{q}\|_{L^{1}}^{\tilde{q}-1}\left\|a_{2}\right\|_{L^{1}}^{\tilde{q}-1}+\|\breve{p}\|_{L^{1}}^{\tilde{q}-1}+\left\|a_{1}\right\|_{L^{1}}^{q-1}\|q\|_{L^{1}}^{q-1}\right) \\
& \times(\|u\|+\|v\|)^{\alpha_{\star}}+\left(\left\|a_{1}\right\|_{L^{1}}^{q-1}\|h\|_{L^{1}}^{q-1}+\left\|a_{2}\right\|_{L^{1}}^{\tilde{q}-1}\|\breve{h}\|_{L^{1}}^{\tilde{q}-1}\right),
\end{aligned}
$$

where

$$
\alpha_{\star}=\max \left\{\alpha_{1}(q-1), \alpha_{2}(q-1), \alpha_{3}(\tilde{q}-1), \alpha_{4}(\tilde{q}-1)\right\} .
$$

If $\|u\|+\|v\|>1$, then

$$
\begin{aligned}
& \frac{\|u\|+\|v\|}{(\|u\|+\|v\|)^{\alpha_{\star}}} \\
& \leq \frac{\max \left\{2^{q-1}, 1\right\}}{(\alpha-\beta)\left(1-\gamma \eta^{\alpha-\beta-1}\right) \Gamma(\alpha)}\left(\left\|a_{1}\right\|_{L^{1}}^{q-1}\|p\|_{L^{1}}^{q-1}+\left\|a_{2}\right\|_{L^{1}}^{\tilde{q}-1}\|\breve{q}\|_{L^{1}}^{\tilde{q}-1}+\left\|a_{2}\right\|_{L^{1}}^{\tilde{q}-1}\|\breve{p}\|_{L^{1}}^{\tilde{q}-1}\right. \\
& \left.\quad+\left\|a_{1}\right\|_{L^{1}}^{q-1}\|q\|_{L^{1}}^{q-1}\right)+\frac{\left(\left\|a_{1}\right\|_{L^{1}}^{q-1}\|h\|_{L^{1}}^{q-1}+\left\|a_{2}\right\|_{L^{1}}^{\tilde{q}-1}\|\breve{h}\|_{L^{1}}^{\tilde{q}-1}\right)}{(\|u\|+\|v\|)^{\alpha_{\star}}}
\end{aligned}
$$

or

$$
\begin{aligned}
& (\|u\|+\|v\|)^{1-\alpha_{\star}} \\
& \quad \leq \frac{\max \left\{2^{q-1}, 1\right\}}{(\alpha-\beta)\left(1-\gamma \eta^{\alpha-\beta-1}\right) \Gamma(\alpha)}\left(\left\|a_{1}\right\|_{L^{1}}^{q-1}\|p\|_{L^{1}}^{q-1}+\left\|a_{2}\right\|_{L^{1}}^{\tilde{q}-1}\|\breve{q}\|_{L^{1}}^{\tilde{q}-1}+\left\|a_{2}\right\|_{L^{1}}^{\tilde{q}-1}\|\breve{p}\|_{L^{1}}^{\tilde{q}-1}\right.
\end{aligned}
$$




$$
\left.+\left\|a_{1}\right\|_{L^{1}}^{q-1}\|q\|_{L^{1}}^{q-1}\right)+\left(\left\|a_{1}\right\|_{L^{1}}^{q-1}\|h\|_{L^{1}}^{q-1}+\left\|a_{2}\right\|_{L^{1}}^{\tilde{q}-1}\|\breve{h}\|_{L^{1}}^{\tilde{q}-1}\right) .
$$

This implies that

$$
\begin{aligned}
\|u\|+\|v\| \leq & {\left[A\left(\left\|a_{1}\right\|_{L^{1}}^{q-1}\|p\|_{L^{1}}^{q-1}+\left\|a_{2}\right\|_{L^{1}}^{\tilde{q}-1}\|\breve{q}\|_{L^{1}}^{\tilde{q}-1}+\left\|a_{2}\right\|_{L^{1}}^{\tilde{q}-1}\|\breve{p}\|_{L^{1}}^{\tilde{q}-1}+\left\|a_{1}\right\|_{L^{1}}^{q-1}\|q\|_{L^{1}}^{q-1}\right)\right.} \\
& \left.+\left(\left\|a_{1}\right\|_{L^{1}}^{q-1}\|h\|_{L^{1}}^{q-1}+\left\|a_{2}\right\|_{L^{1}}^{\tilde{q}-1}\|\breve{h}\|_{L^{1}}^{\tilde{q}-1}\right)\right]^{1-\alpha_{\star}}
\end{aligned}
$$

then

$$
\|u\|+\|v\| \leq[A B+C]^{1-\alpha_{\star}}:=M_{2}
$$

where

$$
\begin{aligned}
& A=\frac{\max \left\{2^{q-1}, 1\right\}}{(\alpha-\beta)\left(1-\gamma \eta^{\alpha-\beta-1}\right) \Gamma(\alpha)}, \\
& B=\left\|a_{1}\right\|_{L^{1}}^{q-1}\|p\|_{L^{1}}^{q-1}+\left\|a_{2}\right\|_{L^{1}}^{\tilde{q}-1}\|\breve{q}\|_{L^{1}}^{\tilde{q}-1}+\left\|a_{2}\right\|_{L^{1}}^{\tilde{q}-1}\|\breve{p}\|_{L^{1}}^{\tilde{q}-1}+\left\|a_{1}\right\|_{L^{1}}^{q-1}\|q\|_{L^{1}}^{q-1}
\end{aligned}
$$

and

$$
C=\left\|a_{1}\right\|_{L^{1}}^{q-1}\|h\|_{L^{1}}^{q-1}+\left\|a_{2}\right\|_{L^{1}}^{\tilde{q}-1}\|\breve{h}\|_{L^{1}}^{\tilde{q}-1} .
$$

As a consequence of Theorem 2.1, the operator $N$ has a fixed point that is a solution of system (1.4). This completes the proof of the theorem.

\section{Multiplicity of positive solutions}

In this section, our goal is to establish positive solutions and multiplicity of solutions for the problem to system (1.4). To this end, first in this section we assume the functions $f, g \in$ $C\left(\mathbb{R}^{2}, \mathbb{R}_{+}\right)$and define the operator on $P$ as $N: P^{2} \rightarrow P^{2}$ to be the completely continuous map $N=\left(N_{1}, N_{2}\right)$ given in the proof of Theorem 3.1. Then (3.5) and (3.6) are equivalent to the fixed point problem

$$
u=N(u), \quad u \in P^{2}
$$

If $v \in P$ and

$$
\begin{aligned}
u_{i}(t)= & \int_{0}^{1} G_{1}(t, s) \varphi_{q}\left(\int_{0}^{s} a_{1}(\tau) v(\tau) d \tau\right) d s \\
& +\frac{\gamma t^{\alpha-1}}{1-\gamma \eta^{\alpha-\beta-1}} \int_{0}^{1} G_{2}(\eta, s) \varphi_{q}\left(\int_{0}^{s} a_{1}(\tau) v(\tau) d \tau\right) d s, \quad i=1,2,
\end{aligned}
$$

and $u_{i}\left(t_{i}\right)=\left\|u_{i}\right\|$, by Lemma 3.2 this implies that, for any $t \in[\rho, 1]$,

$$
\begin{aligned}
u_{i}(t)= & \int_{0}^{1} G_{1}(t, s) \varphi_{q}\left(\int_{0}^{s} a_{1}(\tau) v(\tau) d \tau\right) d s \\
& +\frac{\gamma t^{\alpha-1}}{1-\gamma \eta^{\alpha-\beta-1}} \int_{0}^{1} G_{2}(\eta, s) \varphi_{q}\left(\int_{0}^{s} a_{1}(\tau) v(\tau) d \tau\right) d s
\end{aligned}
$$




$$
\begin{aligned}
u_{i}(t) \geq & \int_{0}^{1} \min G_{1}(t, s) \varphi_{q}\left(\int_{0}^{s} a_{1}(\tau) v(\tau) d \tau\right) d s \\
& +\frac{\gamma t^{\alpha-1}}{1-\gamma \eta^{\alpha-\beta-1}} \int_{0}^{1} G_{2}(\eta, s) \varphi_{q}\left(\int_{0}^{s} a_{1}(\tau) v(\tau) d \tau\right) d s \\
\geq & \int_{0}^{1} \rho^{\alpha-1} G_{1}(1, s) \varphi_{q}\left(\int_{0}^{s} a_{1}(\tau) v(\tau) d \tau\right) d s \\
& +\frac{\gamma \rho^{\alpha-1}}{1-\gamma \eta^{\alpha-\beta-1}} \int_{0}^{1} G_{2}(\eta, s) \varphi_{q}\left(\int_{0}^{s} a_{1}(\tau) v(\tau) d \tau\right) d s \\
\geq & \rho^{\alpha-1}\left[\int_{0}^{1} G_{1}(1, s) \varphi_{q}\left(\int_{0}^{s} a_{1}(\tau) v(\tau) d \tau\right) d s\right. \\
& \left.+\frac{\gamma}{1-\gamma \eta^{\alpha-\beta-1}} \int_{0}^{1} G_{2}(\eta, s) \varphi_{q}\left(\int_{0}^{s} a_{1}(\tau) v(\tau) d \tau\right) d s\right] \\
\geq & \rho^{\alpha-1}\left[\int_{0}^{1} G_{1}(t, s) \varphi_{q}\left(\int_{0}^{s} a_{1}(\tau) v(\tau) d \tau\right) d s\right. \\
& \left.+\frac{\gamma}{1-\gamma \eta^{\alpha-\beta-1}} \int_{0}^{1} G_{2}(\eta, s) \varphi_{q}\left(\int_{0}^{s} a_{1}(\tau) v(\tau) d \tau\right) d s\right] .
\end{aligned}
$$

Hence

$$
u_{i}(t) \geq \rho^{\alpha-1}\left\|u_{i}\right\|, \quad i=1,2 .
$$

Define the cone $P_{i}$ for $i=1,2$ in $P$ by

$$
P_{i}=\left\{u_{i} \in P: u_{i}(t) \geq \rho^{\alpha-1}\left\|u_{i}\right\| \text { for all } t \in[\rho, 1]\right\},
$$

and the product cone $P=P_{1} \times P_{2}$ in $P^{2}$, then $N(P) \subset P$. Before we state our main result, we introduce the following notations: $\alpha_{i}, \beta_{i}>0$ with $\alpha_{i} \neq \beta_{i}$, we let $r_{i}=\min \left\{\alpha_{i}, \beta_{i}\right\}, R_{i}=$ $\max \left\{\alpha_{i}, \beta_{i}\right\} i=1,2$.

$$
\begin{aligned}
& \gamma_{1}=\min \left\{f\left(u_{1}\left(\theta_{1}(t)\right), u_{2}\left(\theta_{1}(t)\right)\right): \rho \leq t \leq 1, \rho^{\alpha-1} \beta_{1} \leq u_{1} \leq \beta_{1}, \rho^{\alpha-1} r_{2} \leq u_{2} \leq R_{2}\right\}, \\
& \gamma_{2}=\min \left\{g\left(u_{1}\left(\theta_{1}(t)\right), u_{2}\left(\theta_{1}(t)\right)\right): \rho \leq t \leq 1, \rho^{\alpha-1} r_{1} \leq u_{1} \leq R_{1}, \rho^{\alpha-1} \beta_{2} \leq u_{2} \leq \beta_{2}\right\}, \\
& \Gamma_{1}=\max \left\{f\left(u_{1}\left(\theta_{1}(t)\right), u_{2}\left(\theta_{1}(t)\right)\right): \rho \leq t \leq 1, \rho^{\alpha-1} \alpha_{1} \leq u_{1} \leq \alpha_{1}, \rho^{\alpha-1} r_{2} \leq u_{2} \leq R_{2}\right\}, \\
& \Gamma_{2}=\max \left\{g\left(u_{1}\left(\theta_{1}(t)\right), u_{2}\left(\theta_{1}(t)\right)\right): \rho \leq t \leq 1, \rho^{\alpha-1} r_{1} \leq u_{1} \leq R_{1}, \rho^{\alpha-1} \alpha_{2} \leq u_{2} \leq \alpha_{2}\right\} .
\end{aligned}
$$

Also, let

$$
A=\min \left\{G_{1}(t, s): \rho \leq t \leq 1,0 \leq s \leq 1\right\}
$$

and

$$
B=\max \left\{G_{1}(t, s): \rho \leq t \leq 1,0 \leq s \leq 1\right\} .
$$


Theorem 4.1 Assume that there exist $\alpha_{i}, \beta_{i}>0$ with $\alpha_{i} \neq \beta_{i}, i=1,2$, such that

$$
\begin{aligned}
& B \Gamma_{1}^{q-1} \leq \alpha_{1}, \quad A \gamma_{1}^{q-1} \geq \beta_{1}, \\
& B \Gamma_{2}^{q-1} \leq \alpha_{2}, \quad A \gamma_{2}^{q-1} \geq \beta_{2} .
\end{aligned}
$$

Then (1.4) has a positive solution $u=\left(u_{1}, u_{2}\right)$ with $r_{i} \leq\left\|u_{i}\right\| \leq R_{i}, i=1,2$, where $r_{i}=$ $\min \left\{\alpha_{i}, \beta_{i}\right\}, R_{i}=\max \left\{\alpha_{i}, \beta_{i}\right\}$. Moreover, the corresponding orbit of $u$ is included in the rectangle $\left[\rho r_{1}, R_{1}\right] \times\left[\rho r_{2}, R_{2}\right]$.

Proof First note that if $u \in P_{r, R}$, then $r_{1} \leq\left\|\mid u_{1}\right\| \leq R_{1}$ and $r_{2} \leq\left\|u_{2}\right\| \leq R_{2}$, and by the definition of $P$,

$$
\left\{\rho^{\alpha-1} r_{1} \leq u_{1}(t) \leq R_{1} \text { and } \rho^{\alpha-1} r_{2} \leq u_{2}(t) \leq R_{2}\right\}
$$

for all $t$, showing that the orbit of $u$ for $t \in[\rho, 1]$ is included in the rectangle $\left[\rho r_{1}, R_{1}\right] \times$ $\left[\rho r_{2}, R_{2}\right]$.

Also, if we know for example that $\left\|u_{1}\right\|=\alpha_{1}$, then

$$
\rho^{\alpha-1} \alpha_{1} \leq u_{1}(t) \leq \alpha_{1} .
$$

We now prove that, for every $u \in P_{r, R}$ and $i \in\{1,2\}$, the following properties hold:

$$
\begin{array}{ll}
\left\|u_{i}\right\|=\alpha_{i} & \text { implies } u_{i} \nprec N_{i}(u), \\
\left\|u_{i}\right\|=\beta_{i} & \text { implies } u_{i} \nsucc N_{i}(u),
\end{array}
$$

guaranteeing the applicability of Theorem 2.2. Indeed, if $\left\|u_{1}\right\|=\alpha_{1}$ and we would have $u_{1} \prec N_{1}(u)$, then

$$
\begin{aligned}
u_{1}(t)< & N_{1}(u)(t) \\
\leq & \int_{0}^{1} \max G_{1}(t, s) \max \left|a_{1}(t) f\left(u\left(\theta_{1}(t)\right), v\left(\theta_{2}(t)\right)\right)\right|^{q-1} d t \\
& +\frac{\gamma t^{\alpha-1}}{1-\gamma \eta^{\alpha-\beta-1}} \int_{0}^{1} G_{2}(\eta, s) \max \left|a_{1}(t) f\left(u\left(\theta_{1}(t)\right), v\left(\theta_{2}(t)\right)\right)\right|^{q-1} d t \\
\leq & B \Gamma_{1}^{q-1}+\Gamma_{1}^{q-1} \frac{\gamma}{1-\gamma \eta^{\alpha-\beta-1}} \int_{0}^{1} G_{2}(\eta, s) \\
\leq & B \Gamma_{1}^{q-1} \\
\leq & \alpha_{1}
\end{aligned}
$$

for all $t$. This yields the contradiction $\alpha_{1}<\alpha_{1}$.

Now, if $\left\|u_{1}\right\|=\beta_{1}$ and $u_{1} \succ N_{1}(u)$, then for $t \in[\rho, 1]$ we obtain

$$
\begin{aligned}
u_{1}(t) & >N_{1}(u)(t) \\
& \geq \int_{0}^{1} \min G_{1}(t, s) \min \left|a_{1}(t) f\left(u\left(\theta_{1}(t)\right), v\left(\theta_{2}(t)\right)\right)\right|^{q-1} d t
\end{aligned}
$$




$$
\begin{aligned}
& +\frac{\gamma t^{\alpha-1}}{1-\gamma \eta^{\alpha-\beta-1}} \int_{0}^{1} G_{2}(\eta, s) \min \left|a_{1}(t) f\left(u\left(\theta_{1}(t)\right), v\left(\theta_{2}(t)\right)\right)\right|^{q-1} d t \\
\geq & A \gamma_{1}^{q-1} \\
\geq & \beta_{1} .
\end{aligned}
$$

Then we deduce that $\beta_{1}>\beta_{1}$, which is a contradiction. Hence (4.2) holds for $i=1$. Similarly, (4.2) is true for $i=2$. By Theorem 2.2, we see that $N$ has at least one fixed point in $P$. Therefore, system (1.4) has at least one positive solution.

Now we study the existence of multiple positive solutions for the systems of fractional boundary value problem with $p$-Laplacian boundary conditions.

$\left(H_{4}\right) f, g$ are positive and increasing, i.e.,

$$
0 \leq u \leq x, 0 \leq v \leq y \quad \text { imply } 0 \leq f(u, v) \leq f(x, y), 0 \leq g(u, v) \leq g(x, y)
$$

We present the following general existence, multiplicity, and localization result.

Theorem 4.2 Let conditions $\left(H_{1}\right)-\left(H_{2}\right)-\left(H_{4}\right)$ hold, and assume that the norm $\|\cdot\|$ is monotone with respect to each cone $P_{i}(i=1,2)$. Moreover, suppose that there exist $\alpha_{i}, \beta_{i}>0$, with $\alpha_{i} \neq \beta_{i}, i=1,2$, such that

$$
\begin{aligned}
& \left\|N_{1}\left(\alpha_{1} \rho^{\alpha-1}, R_{2} \rho^{\alpha-1}\right)\right\|<\alpha_{1}, \quad\left\|N_{2}\left(R_{1} \rho^{\alpha-1}, \alpha_{2} \rho^{\alpha-1}\right)\right\|<\alpha_{2}, \\
& \left\|N_{1}\left(\beta_{1} \rho^{\alpha-1}, 0\right)\right\|>\beta_{1}, \quad\left\|N_{2}\left(0, \beta_{2} \rho^{\alpha-1}\right)\right\|<\beta_{2},
\end{aligned}
$$

where $R_{i}=\max \left\{\alpha_{i}, \beta_{i}\right\}(i=1,2)$.

Then problem (1.4) has at least

(1) one solution $u=\left(u_{1}, u_{2}\right)$ such that $\beta_{i}<\left\|u_{i}\right\|<\alpha_{i}$ for $i=1,2$, if $\alpha_{i}>\beta_{i}$ for $i=1,2$;

(2) two solutions $\left(u_{1}, u_{2}\right)$ and $\left(v_{1}, v_{2}\right)$ such that $\beta_{1}<\left\|u_{1}\right\|<\alpha_{1}, \beta_{2}<\left\|u_{2}\right\|<\alpha_{2}$, $\beta_{1}<\left\|v_{1}\right\|<\alpha_{1}$, and $\left\|v_{2}\right\|<\alpha_{2}$ if $\alpha_{1}>\beta_{1}$ and $\alpha_{2}<\beta_{2}$;

(3) two solutions $\left(u_{1}, u_{2}\right)$ and $\left(v_{1}, v_{2}\right)$ such that $\alpha_{1}<\left\|u_{1}\right\|<\beta_{1}, \alpha_{2}<\left\|u_{2}\right\|<\beta_{2},\left\|v_{1}\right\|<\alpha_{1}$, and $\beta_{2}<\left\|v_{2}\right\|<\alpha_{2}$ if $\alpha_{1}<\beta_{1}$ and $\alpha_{2}>\beta_{2}$;

(4) four solutions $\left(u_{1}, u_{2}\right),\left(v_{1}, v_{2}\right),\left(w_{1}, w_{2}\right)$, and $\left(z_{1}, z_{2}\right)$ such that $\beta_{i}<\left\|u_{i}\right\|<\alpha_{i}, \alpha_{1}<\left\|v_{1}\right\|<\beta_{1}$, and $\left\|v_{2}\right\|<\alpha_{2},\left\|w_{1}\right\|<\alpha_{1}, \alpha_{2}<\left\|w_{1}\right\|<\beta_{2}$, and $\left\|z_{i}\right\|<\alpha_{i}$, if $\alpha_{i}<\beta_{i}$ for $i=1,2$.

Proof We shall apply Theorem 2.3 to the operator $N=\left(N_{1}, N_{2}\right)$ defined as in (3.8) and (3.9). Let us see that it satisfies conditions (2.1)(2.2).

First we prove that

$$
\lambda u_{1} \neq N_{1}(u) \quad \text { for every } u \in k \text { with }\left\|u_{1}\right\|=\alpha_{1},\left\|u_{2}\right\| \leq R_{2} \text { and all } \lambda \geq 1 \text {. }
$$

Indeed, if not,

$$
\lambda\left\|u_{1}\right\|=\lambda \alpha_{1}=\left\|N_{1}(u)\right\| .
$$


From $0 \leq u_{1} \leq \alpha_{1} \rho^{\alpha-1}$ and $0 \leq u_{2} \leq R_{2} \rho^{\alpha-1}$, by $\left(H_{1}\right),\left(H_{4}\right)$ it follows that

$$
\begin{aligned}
& 0 \leq f\left(u_{1}, u_{2}\right) \leq f\left(\alpha_{1} \rho^{\alpha-1}, R_{2} \rho^{\alpha-1}\right) \\
& 0 \leq \varphi_{q}\left(\int_{0}^{s} a_{1}(\tau) f\left(u_{1}, u_{2}\right) d \tau\right) \leq \varphi_{q}\left(\int_{0}^{s} a_{1}(\tau) f\left(\alpha_{1} \rho^{\alpha-1}, R_{2} \rho^{\alpha-1}\right) d \tau d s\right) .
\end{aligned}
$$

By Lemma (3.2) we obtain

$$
0 \leq N_{1}\left(u_{1}, u_{2}\right) \leq N_{1}\left(\alpha_{1} \rho^{\alpha-1}, R_{2} \rho^{\alpha-1}\right)
$$

and the norm of $X$ being monotone,

$$
\left\|N_{1}\left(u_{1}, u_{2}\right)\right\| \leq\left\|N_{1}\left(\alpha_{1} \rho^{\alpha-1}, R_{2} \rho^{\alpha-1}\right)\right\| .
$$

By assumption (4.3),

$$
\left\|N_{1}\left(\alpha_{1} \rho^{\alpha-1}, R_{2} \rho^{\alpha-1}\right)\right\|<\alpha_{1}
$$

so we obtain the contradiction

$$
\lambda \alpha_{1}<\alpha_{1} \text { for some } \lambda \geq 1
$$

Hence (4.5) holds.

Now, we prove that $u_{1} \neq N_{1}(u)+\mu \rho^{\alpha-1}$ for every $u \in P$ with $\left\|u_{1}\right\|=\beta_{1},\left\|u_{2}\right\| \leq R_{2}$ and all $\mu \geq 0$.

Assume the contrary, i.e., $u_{1}=N_{1}(u)+\mu \rho^{\alpha-1}$ for some $u \in P$ with $\left\|u_{1}\right\|=\beta_{1},\left\|u_{2}\right\| \leq$ $R_{2}$ and some $\mu \geq 0$. Then $u_{1}-N_{1}(u) \in P_{1}$, so $0 \leq N_{1}(u) \leq u_{1}$, and the norm of $X$ being monotone

$$
\left\|N_{1}(u)\right\| \leq\left\|u_{1}\right\|=\beta_{1} .
$$

Also, from condition $\left(H_{4}\right), 0 \leq \beta_{1} \rho^{\alpha-1} \leq u_{1}$ and $0 \leq u_{2}$, so we obtain

$$
0 \leq f_{1}\left(\beta_{1} \rho^{\alpha-1}, 0\right) \leq f\left(u_{1}, u_{2}\right)
$$

then by $\left(H_{1}\right)$ we obtain

$$
0 \leq \varphi_{q}\left(\int_{0}^{s} a_{1}(\tau) f_{1}\left(\beta_{1} \rho^{\alpha-1}, 0\right) d \tau\right) \leq \varphi_{q}\left(\int_{0}^{s} a_{1}(\tau) f\left(u_{1}, u_{2}\right) d \tau\right)
$$

and by Lemma 3.2 we conclude $0 \leq N_{1}\left(\beta_{1} \rho^{\alpha-1}, 0\right) \leq N_{1}\left(u_{1}, u_{2}\right)$. Hence, by monotonicity of the norm,

$$
\left\|N_{1}\left(\beta_{1} \rho^{\alpha-1}, 0\right)\right\| \leq\left\|N_{1}\left(u_{1}, u_{2}\right)\right\| .
$$

Now, from (4.6) we have

$$
\left\|N_{1}\left(\beta_{1} \rho^{\alpha-1}, 0\right)\right\| \leq \beta_{1},
$$


which contradicts assumption (4.4). Therefore, conditions (2.1) $-(2.2)$ hold for $\mathrm{i}=1$. Similarly, they can be verified for $i=2$.

\section{Applications}

Example 5.1 Consider the fractional differential equation with advanced argument for $p$-Laplacian:

$$
\left\{\begin{array}{l}
\varphi_{3 / 2}\left(D_{0^{+}}^{5 / 2} u(t)\right)^{\prime}+\frac{t^{-1 / 2}}{4} \frac{t}{1+t}\left(|u(\theta(t))|^{\frac{1}{4}}+|v(\theta(t))|^{\frac{1}{5}}\right)=0, \quad 0<t<1, \\
\varphi_{3 / 2}\left(D_{0^{+}}^{5 / 2} v(t)\right)^{\prime}+\frac{7 t^{-1 / 2}}{2} \frac{t^{2}}{1+t^{2}}\left(13+|v(\theta(t))|^{1 / 4}+|u(\theta(t))|^{\frac{1}{6}}\right)=0, \quad 0<t<1, \\
D_{0^{+}}^{5 / 2} u(0)=u(0)=u^{\prime}(0)=0, \quad D_{0^{+}}^{7 / 6} u(1)=\frac{7}{10} D_{0^{+}}^{7 / 6} u\left(\frac{1}{2}\right), \\
D_{0^{+}}^{5 / 2} v(0)=v(0)=v^{\prime}(0)=0, \quad D_{0^{+}}^{7 / 6} v(1)=\frac{7}{10} D_{0^{+}}^{7 / 6} v\left(\frac{1}{2}\right),
\end{array}\right.
$$

where $\alpha=\frac{5}{2}, \beta=\frac{7}{6}, \eta=\frac{7}{10}, p=\tilde{p}=\frac{3}{2}, q=\tilde{q}=3, a_{1}(t)=\frac{t^{-1 / 2}}{4}, a_{2}(t)=\frac{7 t^{-1 / 2}}{2}, \varphi_{3}\left(\int_{0}^{1} a_{1}(t) d t\right)=$ $\frac{1}{4}, \varphi_{3}\left(\int_{0}^{1} a_{2}(t) d t\right)=\frac{\sqrt{7}}{2}, \alpha_{1} p=\alpha_{3} \tilde{p}=\frac{3}{8} \in(0,1), \alpha_{2} p=\frac{3}{10} \in(0,1), \alpha_{4} \tilde{p}=\frac{3}{12} \in(0,1)$

$$
f(u(\theta(t)), v(\theta(t)))=\frac{t}{1+t}\left(|u(\theta(t))|^{\frac{1}{4}}+|v(\theta(t))|^{\frac{1}{5}}\right), \quad \theta(t)=t^{\gamma}, \gamma \in(0,1),
$$

and

$$
g(u(\theta(t)), v(\theta(t)))=\frac{t^{2}}{1+t^{2}}\left(13+|v(\theta(t))|^{1 / 4}+|u(\theta(t))|^{\frac{1}{6}}\right), \quad \theta(t)=t^{\gamma}, \gamma \in(0,1) .
$$

It is clear that, for all $(t, u, v) \in[0,1] \times \mathbb{R}^{2}$,

$$
\left\{\begin{array}{l}
|f(u, v)| \leq t\left(|u|^{\frac{1}{4}}+|v|^{\frac{1}{5}}\right), \\
|g(u, v)| \leq t^{2}\left(13+|v|^{1 / 4}+|u|^{\frac{1}{6}}\right) .
\end{array}\right.
$$

Hence all the conditions of Theorem 3.1 hold, this implies that problem (5.1) has at least one solution.

Example 5.2 Consider the fractional differential equation with advanced argument for p-Laplacian:

$$
\begin{cases}\varphi_{3 / 2}\left(D_{0^{+}}^{5 / 2} u(t)\right)^{\prime}+\frac{t^{-1 / 2}}{4} f(u(\theta(t)), v(\theta(t)))=0, & 0<t<1, \\ \varphi_{3 / 2}\left(D_{0^{+}}^{5 / 2} v(t)\right)^{\prime}+\frac{7 t^{-1 / 2}}{2} g(u(\theta(t)), v(\theta(t)))=0, & 0<t<1, \\ D_{0^{+}}^{5 / 2} u(0)=u(0)=u^{\prime}(0)=0, & D_{0^{+}}^{7 / 6} u(1)=\frac{7}{10} D_{0^{+}}^{7 / 6} u\left(\frac{1}{2}\right), \\ D_{0^{+}}^{5 / 2} v(0)=v(0)=v^{\prime}(0)=0, \quad D_{0^{+}}^{7 / 6} v(1)=\frac{7}{10} D_{0^{+}}^{7 / 6} v\left(\frac{1}{2}\right), & \end{cases}
$$

where $f, g \in C\left(\mathbb{R}^{2}, \mathbb{R}_{+}\right)$are nondecreasing in $u$ and $v, \theta(t)=t^{\gamma}, \gamma \in(0,1)$. Assume that

$$
\lim _{z \rightarrow \infty} \frac{f(z, z)}{z}=\lim _{z \rightarrow \infty} \frac{g(z, z)}{z}=0
$$

and

$$
\lim _{z \rightarrow 0} \frac{f(z, z)}{z}=\lim _{z \rightarrow \infty} \frac{g(z, z)}{z}=\infty .
$$


From conditions (5.3) and (5.4), we can prove that there exist $\alpha_{1}, \alpha_{2}, \beta_{1}, \beta_{2}>0, \alpha_{1}<\beta_{1}, \alpha_{2}=$ $\beta_{1}$, and $\beta_{2}=\alpha_{1}$ such that

$$
\begin{aligned}
& \frac{f\left(\rho^{\alpha-1} \beta_{1}, \rho^{\alpha-1} \alpha_{1}\right)}{\rho^{\alpha-1} \beta_{1}} \geq \frac{1}{\rho^{\alpha-1} A}, \\
& \frac{g\left(\rho^{\alpha-1} \alpha_{1}, \rho^{\alpha-1} \alpha_{1}\right)}{\rho^{\alpha-1} \beta_{2}} \geq \frac{1}{\rho^{\alpha-1} A}
\end{aligned}
$$

and

$$
\frac{f\left(\alpha_{1}, \beta_{1}\right)}{\alpha_{1}} \leq \frac{1}{B}, \quad \frac{g\left(\beta_{1}, \beta_{1}\right)}{\alpha_{2}} \leq \frac{1}{B} .
$$

Then we set

$$
\begin{aligned}
& r_{i}=\alpha_{1}, \quad R_{i}=\beta_{1} \quad \text { for } i \in\{1,2\}, \\
& \Gamma_{1}=f\left(\alpha_{1}, \beta_{1}\right), \quad \Gamma_{2}=g\left(\beta_{1}, \beta_{1}\right),
\end{aligned}
$$

and

$$
\gamma_{1}=f\left(\rho^{\alpha-1} \beta_{1}, \rho^{\alpha-1} \alpha_{1}\right), \quad \gamma_{2}=g\left(\rho^{\alpha-1} \alpha_{1}, \rho^{\alpha-1} \alpha_{1}\right) .
$$

We concluded that (5.5) and (5.6) guarantee (4.1). Hence, by Theorem 4.2, problem (5.2) has a positive solution.

Since $f, g$ are positive and increasing, we can easily show that

$$
\begin{array}{ll}
\frac{f\left(\rho^{\alpha-1} \beta_{1}, 0\right)}{\beta_{1}} \geq \frac{1}{A}, & \frac{f\left(\rho^{\alpha-1} \alpha_{1}, \rho^{\alpha-1} R_{2}\right)}{\alpha_{1}}<\frac{1}{B}, \\
\frac{g\left(, 0, \rho^{\alpha-1} \beta_{2}\right)}{\beta_{2}} \leq \frac{1}{A}, & \frac{g\left(\rho^{\alpha-1} R_{1}, \alpha_{2} \rho^{\alpha-1}\right)}{\alpha_{2}}<\frac{1}{B} .
\end{array}
$$

Thus conditions (4.3) and (4.4) hold. Then, by Theorem 4.2, problem (5.2) has multiplicity of solutions.

\section{Conclusions}

In this present work, we discussed some existence multiplicity results for system of fractional differential equations, under various assumptions on the right-hand side nonlinearity. The main assumptions on the nonlinearity are the continuity and some NagumoBernstein type growth conditions. We have used fixed point theory in vector metric spaces with general properties from functional analysis. Also the positivity result for a fractional system of differential equations was considered. We hope that this paper can provide contributions to the questions of existence, positivity, and multiplicity of solutions for fractional differential equations on bounded domains. 
Availability of data and materials

All data generated or analysed during this study are included in this published article.

\section{Competing interests}

The authors declare that they have no competing interests.

\section{Authors' contributions}

All authors contributed equally and significantly in writing this article. All authors read and approved the final manuscript.

\section{Author details}

'Laboratory of Mathematics, Sidi-Bel-Abbès University, PoBox 89, 22000, Sidi-Bel-Abbès, Algeria. ${ }^{2}$ Departamento de Estatística, Análise Matemática, e Optimización, Instituto de Matemáticas, Universidade de Santiago de Compostela, Santiago de Compostela, 15782, Spain.

\section{Publisher's Note}

Springer Nature remains neutral with regard to jurisdictional claims in published maps and institutional affiliations.

\section{Received: 17 March 2021 Accepted: 14 July 2021 Published online: 29 July 2021}

\section{References}

1. Abdeljawad, T., Agarwal, R.P., Karapinar, E., Kumari, P.S.: Solutions of the nonlinear integral equation and fractional differential equation using the technique of a fixed point with a numerical experiment in extended $b$-metric space. Symmetry 11(5, $686(2019)$

2. Adigzel, R.S., Aksoy, Ü., Karapinar, E., Erhan, I.: On the solution of a boundary value problem associated with a fractional differential equation. Math. Methods Appl. Sci. (2020). https://doi.org/10.1002/mma.6652

3. Adguzel, R.S., Aksoy, Ü., Karapinar, E., Erhan, I.M.: On the solutions of fractional differential equations via Geraghty type hybrid contractions. Appl. Comput. Math. 20(2), 313-333 (2021)

4. Adguzel, R.S., Aksoy, Ü., Karapinar, E., Erhan, I.M.: Uniqueness of solution for higher-order nonlinear fractional differential equations with multi-point and integral boundary conditions. RACSAM 115, 155 (2021). https://doi.org/10.1007/s13398-021-01095-3

5. Afshari, H., Kalantari, S., Karapinar, E.: Solution of fractional differential equations via coupled fixed point. Electron. J. Differ. Equ. 2015, 286 (2015)

6. Alqahtani, B., Aydi, H., Karapinar, E., Rakočević, V.: A solution for Volterra fractional integral equations by hybrid contractions. Mathematics 7, 694 (2019)

7. Bai, Z., Lü, H.: Positive solutions for a boundary value problem of nonlinear fractional differential equation. J. Math. Anal. Appl. 311, 495-505 (2005)

8. Bolojan-Nica, O., Infante, G., Precup, R.: Existence results for systems with coupled nonlocal initial conditions. Nonlinear Anal. 4, 231-242 (2014)

9. Cabada, A., Wang, G.: Positive solutions of nonlinear fractional differential equations with integral boundary value conditions. J. Math. Anal. Appl. 389, 403-411 (2012)

10. Chai, G.: Positive solutions for boundary value problems of fractional differential equation with $p$-Laplacian. Bound. Value Probl. (2012)

11. Gaul, L., Klein, P., Kempfle, S.: Damping description involving fractional operators. Mech. Syst. Signal Process. 5, 81-88 (1991)

12. Graef, J.R., Henderson, J., Ouahab, A.: Topological Methods for Differential Equations and Inclusions. Monographs and Research Notes in Mathematics Series Profile. CRC Press, Boca Raton (2019)

13. Graef, J.R., Kadari, H., Ouahab, A., Oumansour, A.: Existence results for systems of second-order impulsive differential equations. Acta Math. Univ. Comen. 88, 51-66 (2019)

14. Guo, D., Lakshmikantham, V.: Nonlinear Problems in Abstract Cones. Academic Press, San Diego (1988)

15. Guendouz, C., Lazreg, J.E., Nieto, J.J., Ouahab, A.: Existence and compactness results for a system of fractional differential equations. J. Funct. Spaces 2020, Article ID 5735140 (2020)

16. Henderson, J., Ouahab, A., Slimani, M.: Existence results of system of semilinear discrete equations. Int. J. Difference Equ. 12, 235-253 (2017)

17. Karapinar, E., Fulga, A., Rashid, M., Shahid, L., Aydi, H.: Large contractions on quasi-metric spaces with an application to nonlinear fractional differential equations. Mathematics 7(4), 444 (2019)

18. Kadari, H., Nieto, J.J., Ouahab, A., Oumansour, A.: Implicit first order impulsive differential systems. Int. J. Difference Equ. 15, 429-451 (2020)

19. Lazreg, J.E., Abbas, S., Benchohra, M., Karapinar, E.: Impulsive Caputo-Fabrizio fractional differential equations in b-metric spaces. Open Math. 19, 363-372 (2021)

20. Liang, R., Peng, J., Shen, J.: Double positive solutions for a nonlinear four-point boundary value problem with a p-Laplacian operator. Nonlinear Anal. 68, 1881-1889 (2008)

21. Malki, Z., Berhoun, F., Ouahab, A.: System of boundary random fractional differential equations via Hadamard derivative. Ann. Univ. Paedagog. Crac. Stud. Math. 20, 17-41 (2021)

22. Malinowska, A.B., Torres, D.F.M.: Towards a combined fractional mechanics and quantization. Fract. Calc. Appl. Anal. $15,407-417(2012)$

23. Metzler, F., Schick, W., Kilian, H.G., Nonnenmacher, T.F.: Relaxation in filled polymers: a fractional calculus approach. J. Chem. Phys. 103, 7180-7186 (1995)

24. Miller, K.S., Ross, B.: An Introduction to the Fractional Calculus and Fractional Differential Equations. Wiley, New York (1993)

25. Nasholm, S.P., Holm, S.: On a fractional Zener elastic wave equation. Fract. Calc. Appl. Anal. 16, 26-50 (2013) 
26. Nica, O.: Initial-value problems for first-order differential systems with general nonlocal conditions. Electron. J. Differ. Equ. 2012, 74 (2012)

27. Nica, O., Precup, R.: On the nonlocal initial value problem for first order differential systems. Stud. Univ. Babeş-Bolyai, Math. 56(3), 125-137 (2011)

28. Nieto, J.J., Ouahab, A., Slimani, M.A.: Existence and boundedness of solutions for systems of difference equations with infinite delay. Glas. Mat. 53(73), 123-141 (2018)

29. Precup, R., Rodríguez-López, J.: Multiplicity results for operator systems via fixed point index. Results Math. 74, 25 (2019)

30. Precup, R.: A vector version of Krasnosel'skil's fixed point theorem in cones and positive periodic solutions of nonlinear systems. J. Fixed Point Theory Appl. 2, 141-151 (2007)

31. Su, H., Wei, Z., Wang, B.: The existence of positive solutions for a nonlinear four-point singular boundary value problem with a $p$-Laplacian operator. Nonlinear Anal. 66, 2204-2217 (2007)

32. El-Sayed, A.M.A.: Nonlinear functional differential equations of arbitrary orders. Nonlinear Anal. 33, 181-186 (1998)

33. Su, Y., Feng, Z.: Existence theory for an arbitrary order fractional differential equation with deviating argument. Acta Appl. Math. 118, 81-105 (2012)

34. Tang, X., Yan, C., Liu, Q.: Existence of solutions of two point boundary value problems for fractional $p$-Laplace differential equations at resonance. J. Appl. Math. Comput. 41,119-131 (2013)

35. Torres, F.: Positive solutions for fractional boundary value problem with $p$-Laplacian. Int. J. Math. Anal. 10, 469-484 (2018)

36. Viorel, A.: Contributions to the Study of Nonlinear Evolution Equations. Ph.D. thesis, Babeş-Bolyai University Cluj-Napoca, Department of Mathematics (2011)

37. Kilbas, A.A., Trujillo, J.J.: Differential equations of fractional order: methods, results and problems II. Appl. Anal. 81, 435-493 (2002)

38. Zhao, D., Wang, H., Ge, W.: Existence of triple positive solutions to a class of $p$-Laplacian boundary value problems. J. Math. Anal. Appl. 328, 972-983 (2007)

\section{Submit your manuscript to a SpringerOpen ${ }^{\circ}$ journal and benefit from:}

- Convenient online submission

- Rigorous peer review

- Open access: articles freely available online

- High visibility within the field

- Retaining the copyright to your article

Submit your next manuscript at $\gg$ springeropen.com 